\title{
Temperature and H/D Isotopic Effects in the IR Spectra of the Hydrogen Bond in Solid-State 2-Furanacetic Acid and 2-Furanacrylic Acid
}

\author{
Henryk T. Flakus and Anna Jarczyk-Jędryka \\ Institute of Chemistry, University of Silesia, 9 Szkolna Street, 40-006 Katowice, Poland
}

Correspondence should be addressed to Henryk T. Flakus, flakus@ich.us.edu.pl

Received 1 March 2012; Revised 16 June 2012; Accepted 4 July 2012

Academic Editor: Joanna Sadlej

Copyright ( $) 2012$ H. T. Flakus and A. Jarczyk-Jędryka. This is an open access article distributed under the Creative Commons Attribution License, which permits unrestricted use, distribution, and reproduction in any medium, provided the original work is properly cited.

\begin{abstract}
Polarized IR spectra of 2-furanacetic acid and of 2-furanacrylic acid crystals were measured at $293 \mathrm{~K}$ and $77 \mathrm{~K}$ in the $v_{\mathrm{O}-\mathrm{H}}$ and $v_{\mathrm{O}-\mathrm{H}}$ band frequency ranges. The corresponding spectra of the two individual systems strongly differ, one from the other, by the corresponding band shapes as well as by the temperature effect characterizing the bands. The crystal spectral properties remain in a close relation with the electronic structure of the two different molecular systems. We show that a vibronic coupling mechanism involving the hydrogen bond protons and the electrons on the $\pi$-electronic systems in the molecules determines the way in which the vibrational exciton coupling between the hydrogen bonds in the carboxylic acid dimers occurs. A strong coupling in 2-furanacrylic acid dimers prefers a "tail-to-head-" type Davydov coupling widespread by the $\pi$-electrons. A weak throughspace coupling in 2-furanacetic acid dimers is responsible for a "side-to-side-" type coupling. The relative contribution of each exciton coupling mechanism in the dimer spectra generation is temperature and the molecular electronic structure dependent. This explains the observed difference in the temperature-induced evolution of the compared spectra.
\end{abstract}

\section{Introduction}

Infrared spectroscopy still constitutes a basic tool in the research of the hydrogen bond dynamics. The $\nu_{\mathrm{X}-\mathrm{H}}$ bands measured in the highest frequency range of the mid-infrared attributed to the proton stretching vibrations in $\mathrm{X}-\mathrm{H} \cdots \mathrm{Y}$ hydrogen bonds are the source of wealth data system in this matter. Complex fine structure patterns of these bands are considered as the result of anharmonical coupling mechanisms involving the proton stretching vibrations and other normal vibrations occurring in associated molecular systems, mainly the low-frequency $\mathrm{X} \cdots \mathrm{Y}$ hydrogen bridge stretching vibrational motions [1-5]. The band contour shapes are extremely susceptible on the influences exerted by diverse physical factors, such as changes of temperature, changes in the matter state of condensation, pressure, and solvents [1-5].

Among the contemporary theories of the IR spectra of the hydrogen bond, formed in molecular systems, quantitative theoretical models elaborated for the description of the $v_{\mathrm{X}-\mathrm{H}}$ band generation mechanisms are of the particular importance. There are two most advanced quantitative theoretical models, namely, the "strong-coupling" theory [68] (the elder theory) and the "relaxation" (linear response) theory, the novel model $[9,10]$. Both models are of a purely vibrational nature. Over the last four decades, by using of these theories, IR spectra of diverse hydrogen bond systems have been reproduced satisfactorily. The model calculations concerned quantitative interpretation of spectra of single, isolated hydrogen bonds $[7,11]$, spectra of cyclic dimeric hydrogen bond systems [7, 12-14], and the IR spectra of hydrogen-bonded molecular crystals [15]. Simultaneously, the H/D isotopic effects observed in the spectra of the deuterium-bonded corresponding systems have been interpreted [7-15].

Nevertheless, despite the doubtless successes achieved in this area, when interpreting the hydrogen bond system spectra, it seems that a number of basic theoretical problems still remain unsolved. It also seems that the main source in the understanding of many spectral phenomena characterizing 
systems consisting with a number of mutually coupled hydrogen bonds, in terms of the two different quantitative approaches, is in the early history of these studies. In practice, up to the beginning of the 90 s of the 20th century, these studies were restricted to the interpretation of spectra of a number of very simple hydrogen bond systems, mainly to the spectra of cyclic acetic acid dimers formed in the gaseous phase $[7,12-14]$. The extension of this research over other, more diversified and complex hydrogen bond aggregates allowed us to recognize numerous puzzling spectral effects attributed to these systems. Interpretation of these effects seemed to be beyond the contemporary quantitative theoretical models of the hydrogen bond IR spectra without assuming that some not revealed yet mechanisms codecide in the spectra generation.

For the last decade, spectroscopy in polarized light of hydrogen-bonded molecular crystals has provided key experimental data in this area. By measuring of polarized IR spectra of spatially oriented molecular crystals, characterized by a rich diversity of hydrogen bond arrangements met in their lattices, the most complete information has been be obtained about the coupling mechanisms involving hydrogen bonds in these systems. It appeared that the investigation of spectra of even so simple mutually interacting hydrogen bond aggregates like cyclic dimers (e.g., carboxylic acid dimers) allowed to reveal new H/D isotopic effects, namely, the H/D isotopic self-organization effects. They depend on a nonrandom distribution of protons and deuterons in the crystal lattices of isotopically diluted hydrogen bond systems. These spectral effects may be considered as the manifestation of a new kind of cooperative interactions involving hydrogen bonds, that is, the so-called dynamical cooperative interactions [16-18]. This revealing has emphasized the role of the vibronic coupling between the electronic and the proton vibrational motions taking place in hydrogen bond aggregates, in the generation of the very nature of the hydrogen bond as the natural phenomenon and in the interhydrogen bond interaction mechanisms $[17,18]$.

In the lattices of carboxylic acid crystals, centrosymmetric hydrogen bond dimers, present in the $(\mathrm{COOH})_{2}$ cycles, are frequently met $[19,20]$. These dimers are the bearers of the main crystal spectral properties in the frequency ranges of the $\nu_{\mathrm{O}-\mathrm{H}}$ bands attributed to the proton stretching vibrations. One might expect that regardless of the molecular structure of carboxylic acids in their fragments placed outside the carboxyl groups, the $\nu_{\mathrm{O}-\mathrm{H}}$ band contour shapes should be fairly similar one to the other. This presumption is based on the considerations of the classic vibrational analysis, which predicted that the proton stretching vibrations in these molecules practically do not mix with vibrations of other atomic groups [21]. The experiment learns, however, that spectra of diverse carboxylic acid crystals considerably differ, one from the other, with regard to their $\nu_{\mathrm{O}-\mathrm{H}}$ band contour shapes as well as with regard to the temperature effects measured in the spectra. Qualitatively similar conclusion is valid for the $\nu_{\mathrm{O}-\mathrm{D}}$ bands in the spectra of the deuteriumbonded species [22-27]. Our hitherto estimations, resulting from the comparison of the IR crystalline spectra of diverse carboxylic acid molecular systems, ascribe the differences between the compared spectra in relation to the differences in the electronic structure of carboxylic acid molecules. For instance, $\pi$-electronic systems of aromatic rings or other larger conjugated $\pi$-electronic systems, linked directly to carboxyl groups, strongly change the basic spectral properties of carboxylic acid dimers in comparison with the analogous properties of aliphatic carboxylic acids [2227]. The generation mechanism of these effects still remains unknown.

This paper deals with IR spectra of the hydrogen bond in crystals of two different carboxylic acids, namely, of 2furanacetic acid and 2-furanacrylic acid. In these crystalline systems, associated molecules form hydrogen-bonded cyclic, centrosymmetric dimers (Complete crystallographic data for 2-furanacetic acid and (excluding structure factors) have been deposited at the Cambridge Crystallographic Data Centre under the number CCDC-885823. Copies can be obtained free of charge from CCDC, 12 Union Road, Cambridge CB2 1EZ, U.K. (Fax: Int.+1223-336-033; e-mail: deposit@ccdc.cam.ac.uk)). The crystallographic data for 2furanacrylic acid can be found in $[28,29]$. Molecules of these two individual molecular systems differ, one from the other, by their electronic structures. In the latter case, the carboxyl groups are directly linked to the large $\pi$-electronic systems. In the 2-furanacetic acid crystal case, methylene groups separate the hydrogen bonds, formed by the associated carboxyl groups, from the $\pi$-electronic system of furan rings.

The aim of the study reported in this paper was to provide new arguments of experimental nature about the role of the electronic structures of carboxylic acid molecules in the generation of IR spectra of cyclic hydrogen bond dimers. The investigation results presented constitute a part of results obtained in the frames of a wider project, which also assumed measuring of crystalline spectra of other carboxylic acids, mainly of furan and thiophene derivatives. Our choice of these model molecular systems was strongly supported by advantageous well-developed $\nu_{\mathrm{O}-\mathrm{H}}$ and $\nu_{\mathrm{O}-\mathrm{D}}$ band contour shapes in the IR spectra of these systems. We expected that the quantitative analysis of the polarized IR spectra of 2-furanacetic acid and 2-furanacrylic acid crystals and also of the spectra of relative carboxylic acid crystals should provide new arguments for the formulation of a new theoretical approach for the description of the hydrogen bond dimer spectra. The understanding of the temperature effects and the generation mechanism of the intensity distribution patterns in the $\nu_{\mathrm{O}-\mathrm{H}}$ and $\nu_{\mathrm{O}-\mathrm{D}}$ bands in the spectra of diverse carboxylic acid crystals are of the particular interest and importance in this project.

\section{X-Ray Structures of 2-Furanacetic Acid and 2-Furanacrylic Acid}

Crystals of 2-furanacetic acid are monoclinic and the spacesymmetry group is $\mathrm{P} 2{ }_{1} / \mathrm{c}, Z=4$. The lattice constants at $100 \mathrm{~K}: a=13.0525(4) \AA ; b=4.85360(10) \AA ; c=9.4107(3) \AA$, $\beta=103.832(3)^{\circ}$. In a unit cell four translationally nonequivalent molecules form two plain centrosymmetric cyclic hydrogen-bonded dimers (Complete crystallographic data for 2-furanacetic acid (excluding structure factors) have been 


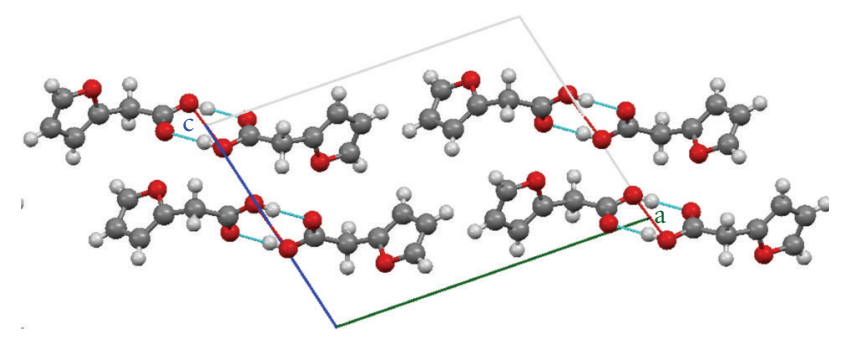

Figure 1: The X-ray structure of 2-furanacetic acid crystal. Projection of the lattice onto the " $a c$ " plane.

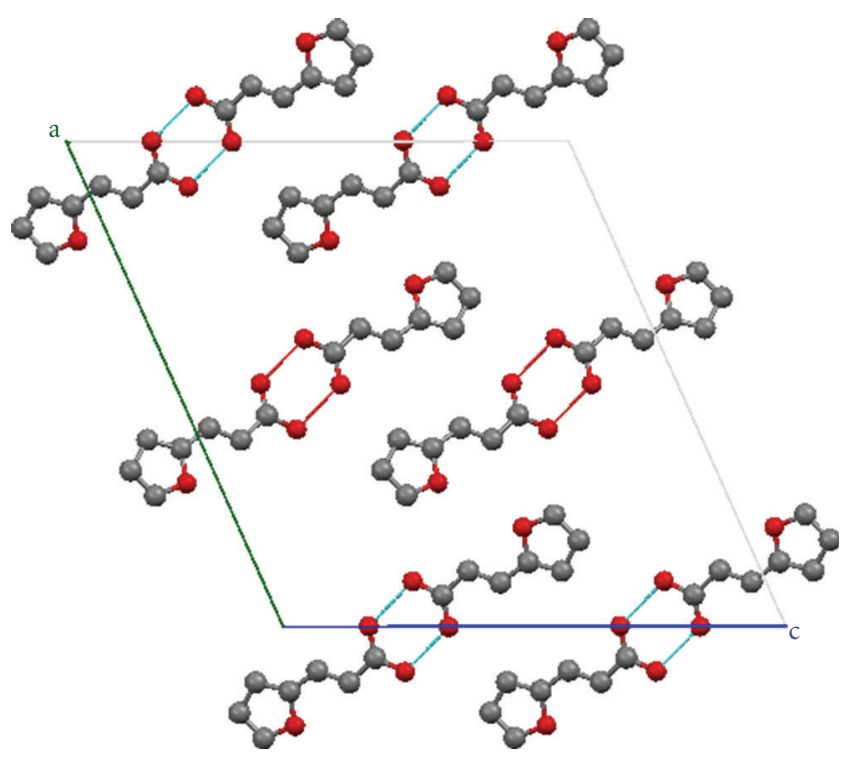

Figure 2: The X-ray structure of 2-furanacrylic acid crystal. Projection onto the "ac" plane.

deposited at the Cambridge Crystallographic Data Centre under the number CCDC-885823). The molecules of 2furanacetic acid in the lattice are linked together by the O$\mathrm{H}$. . O hydrogen bonds, forming centrosymmetric dimers. A view of the crystal lattice of 2-furanacetic acid is shown in Figure 1.

Crystals of 2-furanacrylic acid are also monoclinic, the space-symmetry group is $\mathrm{C} 2 / \mathrm{c}$ and $\mathrm{Z}=8$. The unit cell parameters are $a=18.975 \AA ; b=3.843 \AA$; $c=20.132 \AA, \beta=$ $113.9^{\circ}$. The molecules of 2-furanacrylic acid in the lattice are linked together by the $\mathrm{O}-\mathrm{H}$. . O hydrogen bonds, forming cyclic approximately centrosymmetric dimers [28, 29]. The $\mathrm{X}$-ray structure of 2-furanacrylic acid crystals is shown in Figure 2.

\section{Experimental}

2-Furanacetic acid $\left(\mathrm{C}_{4} \mathrm{H}_{3} \mathrm{O}-\mathrm{CH}_{2}-\mathrm{COOH}\right)$ and 2-furanacrylic acid $\left(\mathrm{C}_{4} \mathrm{H}_{3} \mathrm{O}-\mathrm{CH}=\mathrm{CH}-\mathrm{COOH}\right)$ used for our studies were the commercial substance (Sigma-Aldrich). 2-furanacetic acid was employed without further purification, while 2furanacetic acid was purified by crystallization from its acetone solution. The $d_{1}$ deuterium derivatives of the compounds $\left(\mathrm{C}_{4} \mathrm{H}_{3} \mathrm{O}-\mathrm{CH}_{2}-\mathrm{COOD}\right.$ and $\mathrm{C}_{4} \mathrm{H}_{3} \mathrm{O}-\mathrm{CH}=\mathrm{CH}-$ COOD) were obtained by evaporation of $\mathrm{D}_{2} \mathrm{O}$ solution of each compound at room temperature and under reduced pressure. It was found that the deuterium exchange rate for the $\mathrm{COOH}$ groups varied from 60 to $90 \%$ and from 70 to $90 \%$ for different samples, respectively.

Crystals suitable for further spectral studies were obtained by melting solid samples between two closely compressed spaces $\mathrm{CaF}_{2}$ windows, followed by a very slow cooling of the liquid film. By that means, reasonably thin crystals could be received, characterized by their maximum absorbance at the $v_{\mathrm{O}-\mathrm{H}}$ band frequency range near to 0.5 at room temperature. From the crystalline mosaic, adequate monocrystalline fragments, having dimensions of at least $2 \times 2 \mathrm{~mm}$, were selected and then spatially oriented with the help of a polarization microscope. It was found that in each system case the crystals most frequently developed the " $a c$ " crystalline face. These crystals were selected to the experiment by use of a thin, tin plate diaphragm with a $1.5 \mathrm{~mm}$ diameter hole, and then IR spectra of these crystalline fragments were measured by a transmission method. Spectral experiments were accomplished at room temperature and also at the temperature of liquid nitrogen, using polarized IR radiation. In each measurement, two different, mutually perpendicular orientations of the incident beam electric field vector " $E$ " were applied, with respect to the developed face of the crystal lattice. The solid-state polarized spectra were measured with a resolution of $2 \mathrm{~cm}^{-1}$, for the normal incidence of the IR radiation beam with respect to the crystalline face. The IR spectra were measured with the Nicolet Magna 560 FT-IR spectrometer. Measurements of the spectra were repeated for ca. 8 crystals of each isotopomer of an individual compound. Spectra were recorded in a similar manner for the deuterium derivatives.

The Raman spectra of polycrystalline samples of 2furanacetic acid and 2-furanacrylic acid were measured at room temperature with the use of the Bio-Rad FTS-175C FTIR spectrometer at the $1 \mathrm{~cm}^{-1}$ resolution.

\section{Results}

The preliminary experimental studies of spectral properties of 2-furanacetic acid and 2-furanacrylic acid based on the measurements in $\mathrm{CCl}_{4}$ solution in the frequency range of the $\nu_{\mathrm{O}-\mathrm{H}}$ proton stretching vibration bands. The results are shown in Figure 3.

In Figure 4 are shown the $\nu_{\mathrm{O}-\mathrm{H}}$ bands from the IR spectra of the polycrystalline acid samples in $\mathrm{KBr}$ pellets, measured at $298 \mathrm{~K}$ and $77 \mathrm{~K}$, and in Figure 5 the $\nu_{\mathrm{O}-\mathrm{D}}$ bands spectra of the deuterium derivatives samples in the same conditions. The comparatively wealth spectrum of $\nu_{\mathrm{O}-\mathrm{H}}$ and $\nu_{\mathrm{O}-\mathrm{D}}$ bands for 2-furanacrylic acid molecules may be predictable, based on earlier results for cinnamic acid crystals [24], while the $\nu_{\mathrm{O}-\mathrm{H}}$ and $\nu_{\mathrm{O}-\mathrm{D}}$ bands for 2-furanacetic acid crystals are relatively poorer, similarly as in the phenylacetic acid crystal case [25].

Polarized IR spectra of the two crystalline systems measured at the room temperature in the $\nu_{\mathrm{O}-\mathrm{H}}$ band frequency 


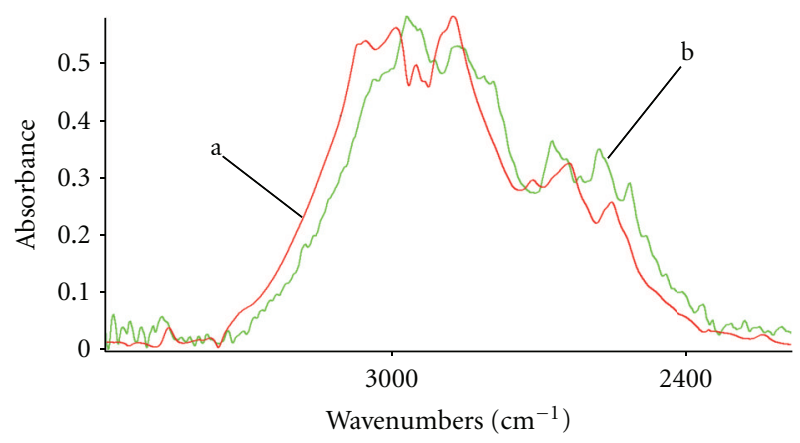

FIgURE 3: The $\nu_{\mathrm{O}-\mathrm{H}}$ band in the IR spectra of (a) 2-furanacetic acid and (b) 2-furanacrylic acid in $\mathrm{CCl}_{4}$ solution.

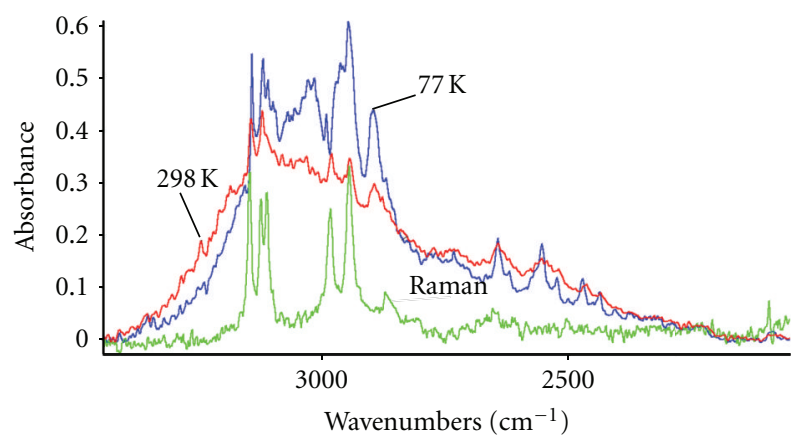

(a)

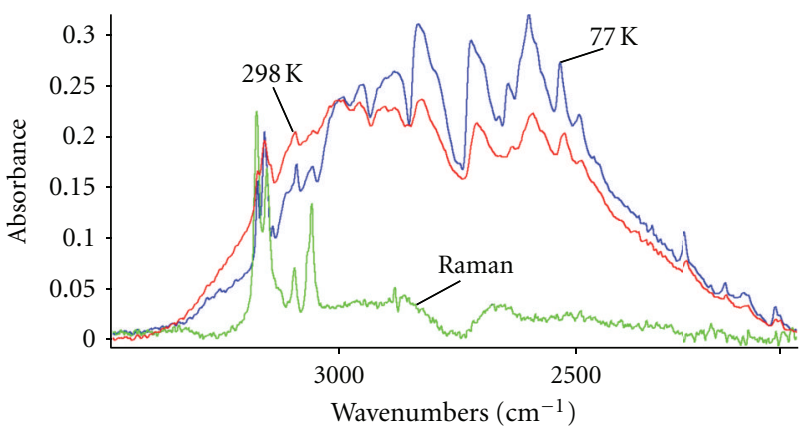

(b)

FIGURE 4: The $\nu_{\mathrm{O}-\mathrm{H}}$ bands in the IR spectra of polycrystalline samples of (a) 2-furanacetic acid and (b) 2-furanacrylic acid, dispersed in $\mathrm{KBr}$ pellets. Temperature effect in the spectra. The Raman spectra measured for polycrystalline samples of the compounds at room temperature are also shown.

range are presented in Figure 6, whereas the corresponding low-temperature spectra are shown in Figure 7.

The corresponding spectra of isotopically diluted crystals recorded in the $\nu_{\mathrm{O}-\mathrm{D}}$ band range are shown in Figures 8 and 9.

The temperature effect in the crystalline spectra in the most intense polarized components of the $\nu_{\mathrm{O}-\mathrm{H}}$ bands is shown in Figure 10 and in the $\nu_{\mathrm{O}-\mathrm{D}}$ bands is given in Figure 11.

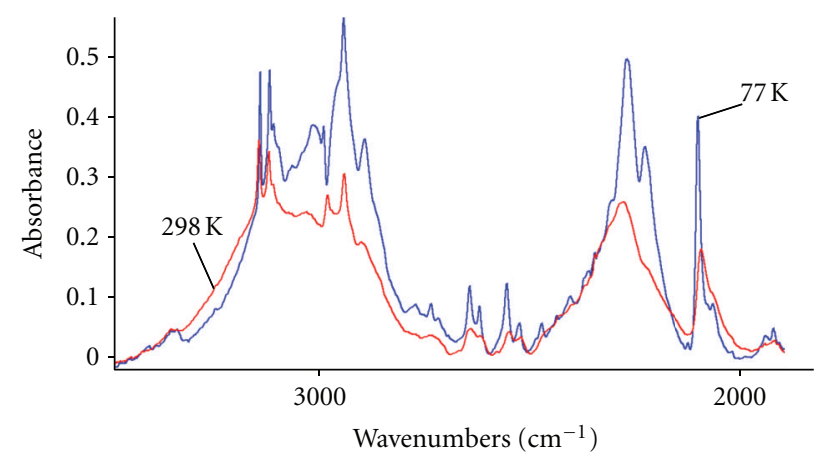

(a)

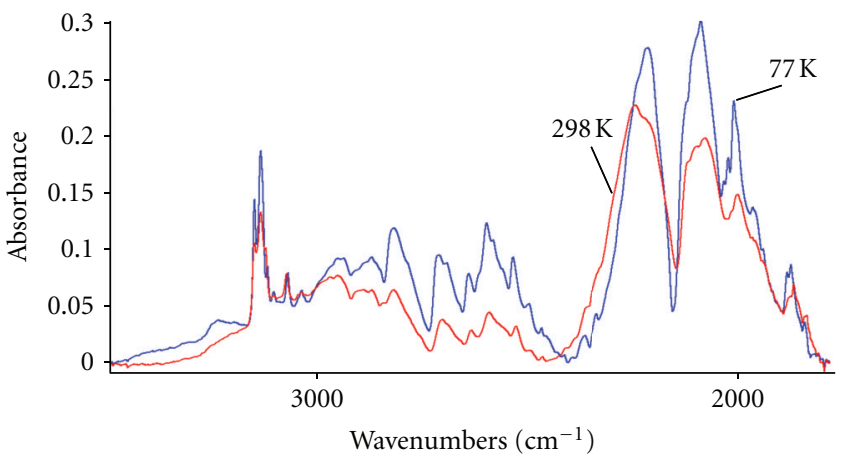

(b)

FIGURE 5: The $\nu_{\mathrm{O}-\mathrm{D}}$ bands in the IR spectra of polycrystalline samples of (a) $d_{1}-2$-furanacetic acid (ca. $45 \% \mathrm{H}$ and $55 \% \mathrm{D}$ ) and (b) $d_{1}$-2-furanacrylic acid (ca. $20 \% \mathrm{H}$ and $80 \% \mathrm{D}$ ) dispersed in $\mathrm{KBr}$ pellets. Temperature effect in the spectra.

\section{Isotopic Dilution Effects in the Crystalline IR Spectra}

On comparing the spectra in Figures 3 and 6-9, it can be noticed that the replacement of the major part of the hydrogen bond protons by deuterons changed the dichroic properties in the "residual" $\nu_{\mathrm{O}-\mathrm{H}}$ band substantially. The band shapes no longer depended on the crystal orientation investigated and resembled the spectrum measured for the $\mathrm{CCl}_{4}$ solution of the compounds. Regardless of the increase in the rates of deuterium substitution in the samples, the "residual" $\nu_{\mathrm{O}-\mathrm{H}}$ band still retained its "dimeric" character. This is due to the fact that the hydrogen-bonded dimeric spectrum measured in the "residual" $\nu_{\mathrm{O}-\mathrm{H}}$ band range is still under the influence of the interhydrogen bond vibrational exciton interactions occurring within each individual carboxylic acid dimer [22-27].

The unusual properties of the "residual" $\nu_{\mathrm{O}-\mathrm{H}}$ bands have proved that the distribution of protons and deuterons between the hydrogen bonds of the isotopically diluted crystalline samples is nonrandom and in an individual dimer the coexistence of two identical hydrogen isotope atoms, proton or deuterons, is preferred. As a result, the interhydrogen bond exciton interactions still occur in each dimeric system and consequently the "residual" $\nu_{\mathrm{O}-\mathrm{H}}$ bands retain their "dimeric" properties. These spectral effects, that is, the so-called H/D isotopic "self-organization" effects, are 


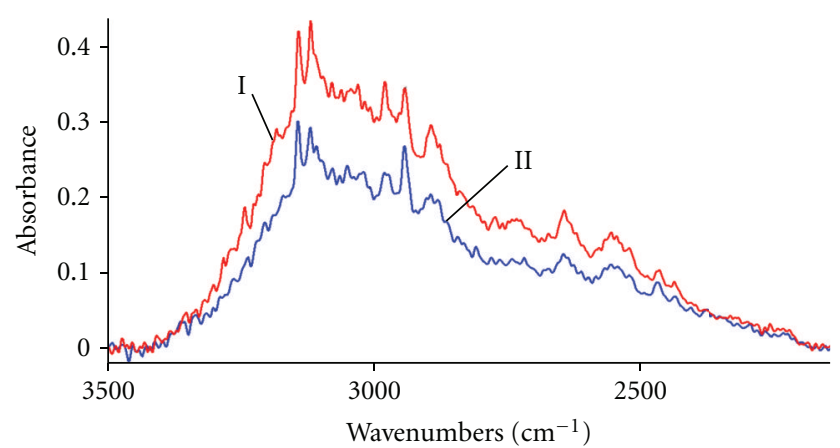

(a)

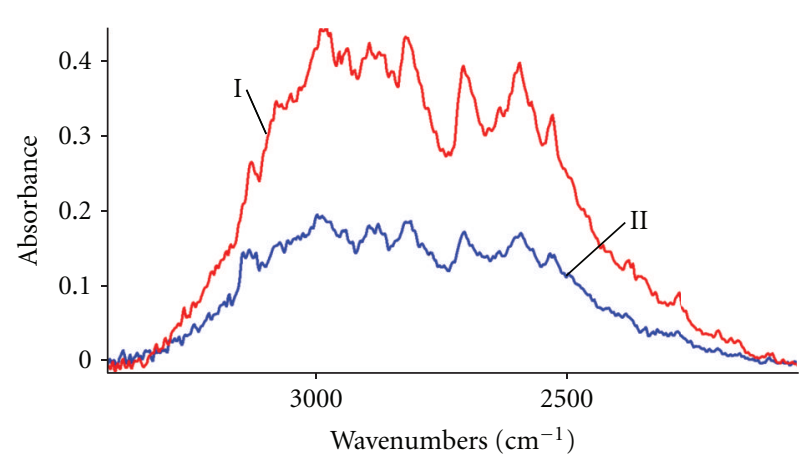

(b)

FIGURE 6: Polarized IR spectra of 2-furanacetic acid and 2-furanacrylic acid crystals measured at room temperature in the $\nu_{\mathrm{O}-\mathrm{H}}$ band frequency range for the IR radiation of the normal incidence with respect to the " $a c$ " crystal faces. (a) 2-furanacetic acid crystal. (I) The electric field vector $E$ of the incident beam of IR radiation parallel to the $a$-axis (II) The E vector parallel to the $c^{*}$-axis (the $c^{*}$-symbol denotes the vector in the reciprocal lattice). (b) 2-furanacrylic acid crystal. (I) The electric field vector E parallel to the $c$-axis. (II) The E vector parallel to the $a^{*}$-axis.

the attribute of the "dynamical cooperative interactions" involving hydrogen bonds in the dimers [16-18].

In the case of high excess of protons in the crystals qualitatively similar spectral effects can be identified in the "residual" $\nu_{\mathrm{O}-\mathrm{D}}$ bands, located in the range of $1900-2300 \mathrm{~cm}^{-1}$, as those observed in the "residual" $\nu_{\mathrm{O}-\mathrm{H}}$ bands. In the low concentration of deuterons, the "residual" $v_{\mathrm{O}-\mathrm{D}}$ bands still retain the characteristic linear dichroic effects accompanying them (see Figures 4-9). For the two compared "residual" bands, $\nu_{\mathrm{O}-\mathrm{H}}$ and $\nu_{\mathrm{O}-\mathrm{D}}$, not only the linear dichroic but also the temperature effects appear to be similar to the corresponding effects measured in the spectra of isotopically neat crystals.

This property results from the "dynamical cooperative interactions" in the hydrogen-bonded systems which lead to the appearance of the so-called H/D isotopic selforganization effects in the hydrogen bond IR spectra $[17,18]$. The source of these nonconventional interactions in the hydrogen bond dimers is a vibronic coupling mechanism involving the totally symmetric proton stretching vibrations and the electronic motions in the systems $[17,18]$. According to the theory of the "dynamical cooperative interactions," the

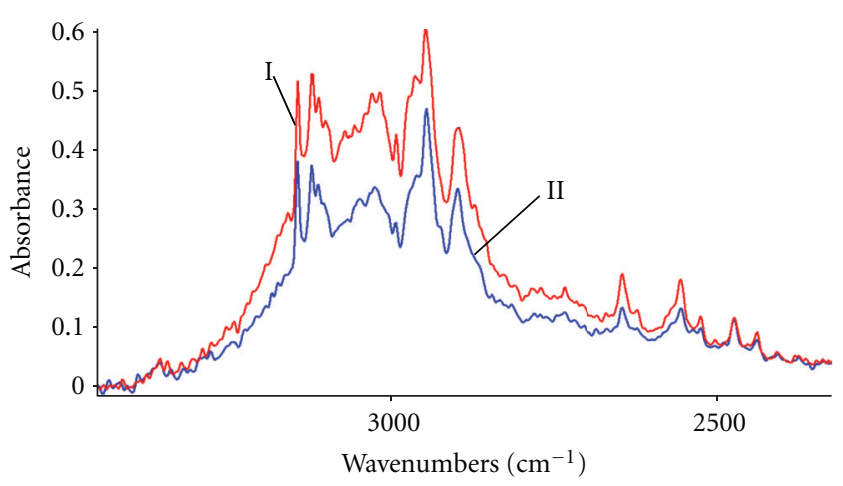

(a)

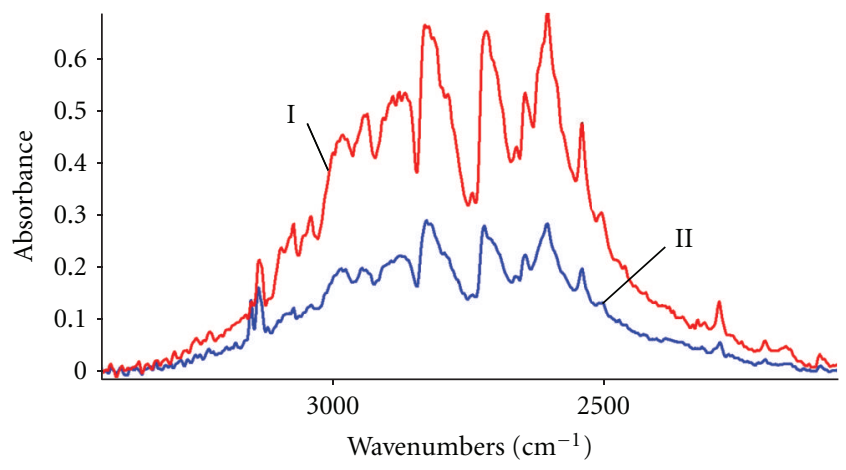

(b)

FIGURE 7: Polarized IR spectra of (a) 2-furanacetic acid and (b) 2-furanacrylic acid crystals measured at $77 \mathrm{~K}$ in the $\nu_{\mathrm{O}-\mathrm{H}}$ band frequency range. (a) 2-furanacetic acid crystal. (I) The electric field vector E parallel to the $a$-axis. (II) The $E$ vector parallel to the $c *$ axis. (b) 2-furanacrylic acid crystal. (I) The electric field vector $E$ parallel to the $c$-axis. (II) The E vector parallel to the $a^{*}$-axis.

symmetric hydrogen bond dimers of the HH or DD-type, with identical hydrogen isotope atoms, are thermodynamically more stable than the non-symmetric dimers of the HD type. The distribution of the $\mathrm{HH}$ - or DD-type dimers in the lattice sites is random. The energy difference between the two forms of dimers, the $\mathrm{HH}$ and the HD types was estimated as approximately equal to $1.5 \mathrm{kcal} /$ mole of the dimers. Therefore, the relative concentration of the HD-type dimers is negligibly low and practically nondetectable with the use of the IR spectroscopic methods [16-18].

From the experimental studies presented in Figures 3-11 it also results that hydrogen-bonded cyclic centrosymmetric dimers are the bearers of the crystal spectral properties, since the inter-dimer vibrational exciton interactions are negligibly small.

\section{Model}

6.1. Carboxylic Acid Dimers the Basic Idea. The problem of the quantitative theoretical treatment of the spectral properties of systems composed with mutually interacting hydrogen bonds still constitute a real challenge in the area of the hydrogen bond research. There are still many problems to solve in this matter, since even the most advanced theories, 


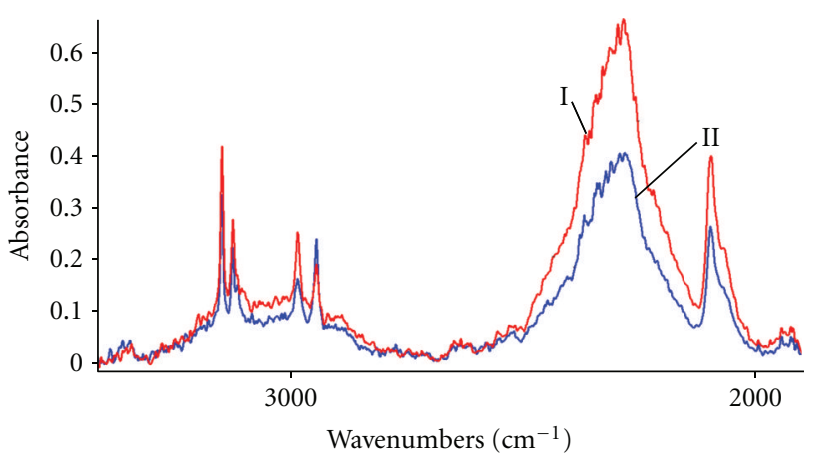

(a)

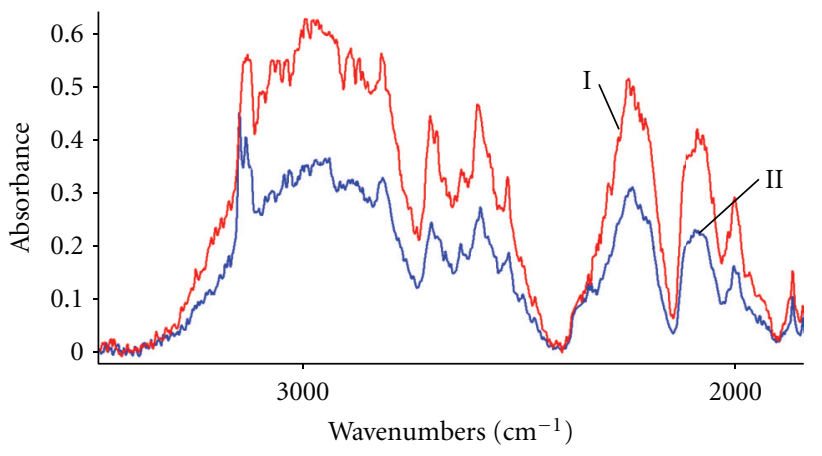

(b)

FIGURE 8: Polarized IR spectra of (a) $d_{1}$-2-furanacetic acid and (b) $d_{1}$-2-furanacrylic acidcrystals measured at room temperature in the $\nu_{\mathrm{O}-\mathrm{D}}$ band frequency range. (a) 2-furanacetic acid crystal (ca. 10\% $\mathrm{H}$ and $90 \% \mathrm{D}$ ). (I) The electric field vector $E$ parallel to the $a$-axis, (II) The $E$ vector parallel to the $c^{*}$-axis (b) 2 -furanacrylic acid crystal (ca. $65 \% \mathrm{H}$ and $35 \% \mathrm{D}$ ). (I) The electric field vector $E$ parallel to the $c$-axis, (II) The E vector parallel to the $a^{*}$-axis.

elaborated for the description of the IR spectra of hydrogen bond systems, are unable to reliably explain a number of effects observed in the dimeric spectra. Despite of spectacular achievements in the quantitative description of the intensity distribution in the $v_{\mathrm{X}-\mathrm{H}}$ bands, which are the attribute of the proton stretching vibrations in the $\mathrm{X}-\mathrm{H}$ - . Y bridges and in the description of the H/D isotopic effects, the understanding of temperature effects in the spectra seems to be totally incomplete.

Cyclic hydrogen bond dimers, formed by associated carboxyl groups of diverse carboxylic acid molecules, are the most frequently studied model systems investigated in this research area. They exhibit some unusual spectral properties in IR connected with the highly abnormal thermal evolution of the $\nu_{\mathrm{O}-\mathrm{H}}$ and $\nu_{\mathrm{O}-\mathrm{D}}$ band contour shapes. One could expect that the hydrogen bond spectra of diverse carboxylic acid dimers, measured in the $\nu_{\mathrm{O}-\mathrm{H}}$ and $\nu_{\mathrm{O}-\mathrm{D}}$ band frequency ranges, should be fairly similar one to another due to the identical structural units of the molecular dimers, namely, the $(\mathrm{COOH})_{2}$ rings, in which two hydrogen bonds exist forming hydrogen bond dimers. However, on comparison of the crystalline spectra of diverse carboxylic acids, a considerable variation degree of the analyzed band contour shapes can be found. This fact undoubtedly remains in a close

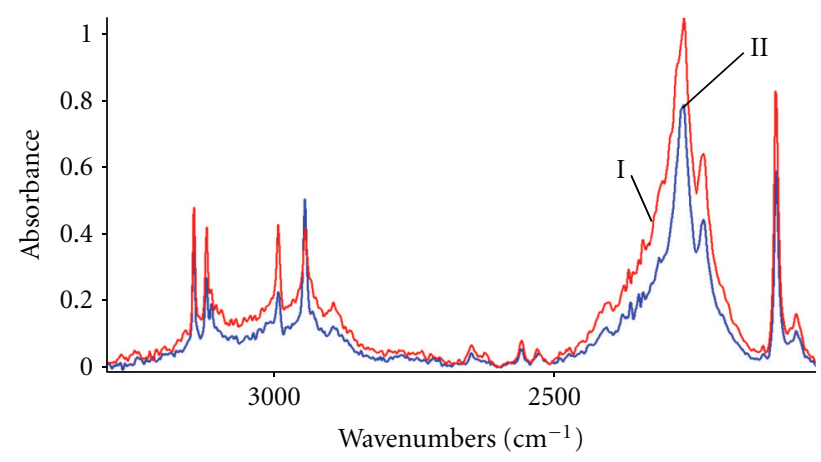

(a)

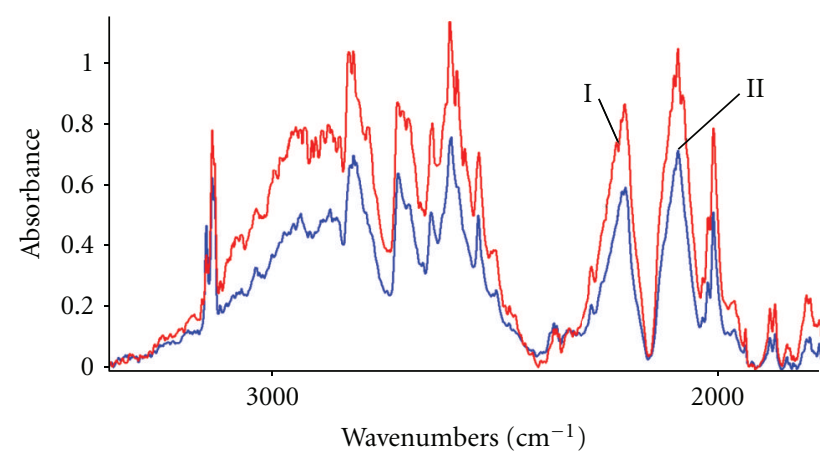

(b)

FIgURE 9: Polarized IR spectra of (a) $d_{1}$-2-furanacetic acid and (b) $d_{1}$-2-furanacrylic acid crystals measured at $77 \mathrm{~K}$ in the $\nu_{\mathrm{O}-\mathrm{D}}$ band frequency range. (a) 2-Furanacetic acid crystal (ca. 10\% $\mathrm{H}$ and $90 \%$ D). (I) The electric field vector $E$ parallel to the $a$-axis, (II) The $E$ vector parallel to the $c^{*}$-axis. (b) 2-Furanacrylic acid crystal (ca $65 \%$ $\mathrm{H}$ and $35 \% \mathrm{D})$. (I) The electric field vector $E$ parallel to the c-axis, (II) The E vector parallel to the $a^{*}$-axis.

connection with differences in the electronic structures of diverse carboxylic acid molecules. Simultaneously, these spectra strongly differ, one from the other, by temperature effects characterizing them. Also these effects undoubtedly remain in a close relation with the electronic structures of the associating molecules. The basic experimental facts supporting the hypothesis given above are presented in the following.

6.2. Electronic Structure of Carboxylic Acid Molecules versus the Temperature Effects in Their Crystalline IR Spectra. Based on our previous studies, at this point, let us summarize the basic properties of the $\nu_{\mathrm{O}-\mathrm{H}}$ bands in the IR spectra of the hydrogen bond cyclic dimers formed by diverse carboxylic acid molecules, in relation to their electronic structures.

(a) In the case of carboxylic acid molecules in which the aliphatic fragments are connected directly with carboxyl groups (e.g., aliphatic monocarboxylic acids [11-13, 30, 31] and dicarboxylic acids [22]), the $\nu_{\mathrm{O}-\mathrm{H}}$ bands are characterized by different intensity distribution patterns, when compared with the corresponding band properties in the IR spectra of arylcarboxylic acids [23, 26]. In the first case, 


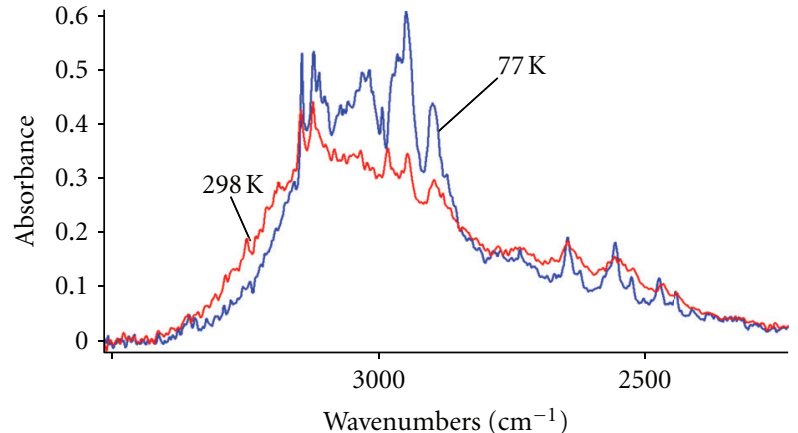

(a)

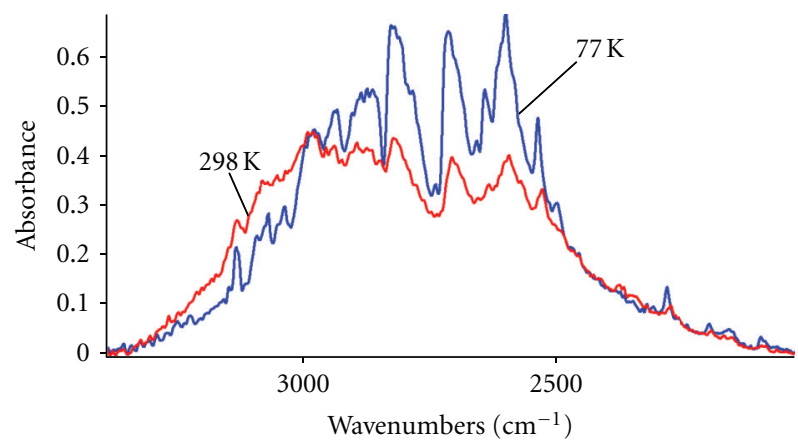

(b)

FIgURE 10: The $\nu_{\mathrm{O}-\mathrm{H}}$ bands in the IR spectra of monocrystalline samples of (a) 2-furanacetic acid and (b) 2-furanacrylic acid. Temperature effect in the spectra.

the higher-frequency branch of the $\nu_{\mathrm{O}-\mathrm{H}}$ band is more intense in relation to the intensity of the lowerfrequency band branch.

(b) In the case of hydrogen-bonded molecular systems, in which carboxyl groups are directly linked to $\pi$ electronic systems (e.g., arylcarboxylic $[23,26]$ and arylacrylic acids [24]), the $\nu_{\mathrm{O}-\mathrm{H}}$ band contours are a "mirror reflection" of the band shapes of systems from the point "a." In this case, the lower-frequency branch of the band is the most intense one. Similar property characterizes spectra of carboxylic acids with other large $\pi$-electronic systems in their molecules, for example, cinnamic acid [24], 2-naphthoic acid [26], and 1-naphthylacrylic acid [32].

(c) For other carboxylic acids, in which aromatic radicals are separated from carboxyl groups by fragments of aliphatic hydrocarbon chains (e.g., arylacetic acid $[25,27]$ and styrylacetic acid [33]), the $\nu_{\mathrm{O}-\mathrm{H}}$ band contour shapes are fairly similar to the corresponding band characteristics from the point "a," that is, to the corresponding spectra of aliphatic monocarboxylic acids [30, 31] and dicarboxylic acids [22]).

The $\nu_{\mathrm{O}-\mathrm{H}}$ bands in the spectra of the hydrogen bond of carboxylic acid crystals from the "a" and "c" groups, measured at room temperature, are characterized by relatively low intensity of the lower-frequency branch of he band in comparison with the higher-frequency band branch

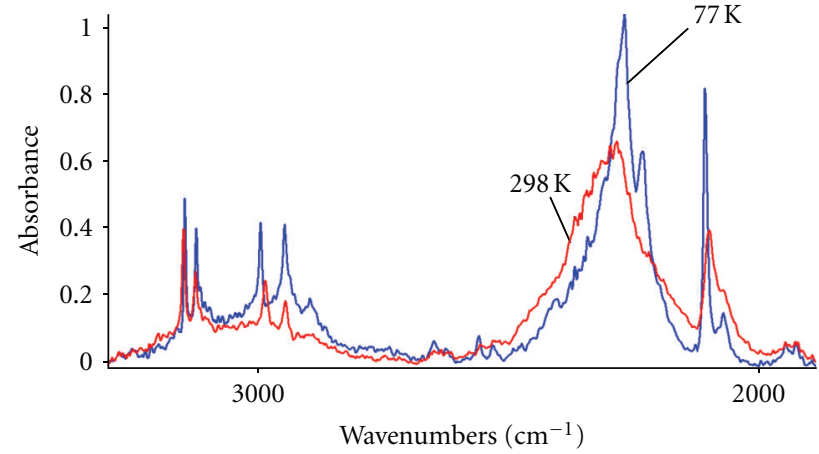

(a)

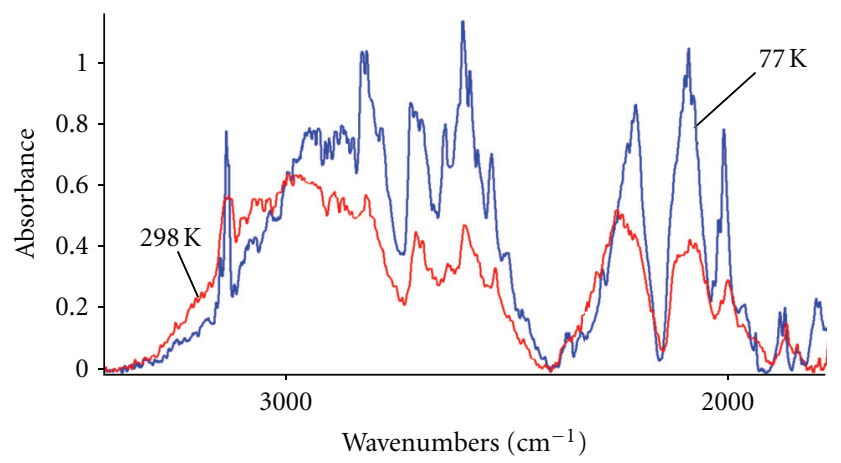

(b)

FIGURE 11: The $v_{\mathrm{O}-\mathrm{D}}$ bands in the IR spectra of monocrystalline samples of (a) $d_{1}$-2-furanacetic acid (ca 10\% $\mathrm{H}$ and $90 \% \mathrm{D}$ ). and (b) $d_{1}$-2-furanacrylic acid (ca $65 \% \mathrm{H}$ and $35 \% \mathrm{D}$ ). Temperature effect in the spectra.

intensity. On the decrease of temperature to $77 \mathrm{~K}$, only a relatively small growth of the relative intensity of the lower-frequency branch of each band can be observed. This band branch still remains of the lower intensity in the lowtemperature spectra.

In the case "b," even at room temperature spectra, the $\nu_{\mathrm{O}-\mathrm{H}}$ bands exhibit relatively high intensity of their lowerfrequency branch in relation to the higher-frequency branch. On the temperature decrease up to $77 \mathrm{~K}$, a considerable growth of the relative intensity of the lower-frequency branch of each analyzed band can be observed. As the result of the band contour thermal evolution, in the low-temperature spectra of carboxylic acid crystals of this group the lowerfrequency branch is of the dominant intensity in the bands.

According to the "state-of-art" in our contemporary knowledge about the quantitative description of the IR spectra of the hydrogen bond in carboxylic acid dimers, the following interpretation of the $\nu_{\mathrm{O}-\mathrm{H}}$ band generation mechanisms seemed to be valid: the lower-frequency branch of the $\nu_{\mathrm{O}-\mathrm{H}}$ band is generated by the transition occurring to the Ag-symmetry excited state of the totally symmetric proton stretching vibrations in the dimers. This transition, forbidden by the symmetry rules, becomes allowed via a vibronic mechanism, which is a kind of reverse of the familiar Herzberg-Teller mechanism, originally responsible for the promotion of forbidden electronic transitions in UV spectra 
of aromatic hydrocarbons [34]. Within this approach of the reverse Herzberg-Teller vibronic coupling mechanism, electronic properties of single hydrogen bonds themselves, as well as electronic properties of the whole associated molecules and the proton vibration anharmonicity, are responsible for the magnitude of the forbidden transition promotion effects in the dimeric spectra [35]. The promotion mechanism determines a unique property of centrosymmetric hydrogen bond dimeric system. This effect found no counterpart in the vibrational spectroscopy of single centrosymmetric molecules.

On the other hand, the higher-frequency spectral branch of the band corresponds with the symmetry-allowed transition to the Au-state of the nontotally symmetric proton vibrations in the centrosymmetric hydrogen bond dimers. One should expect that the higher-frequency branch of the $\nu_{\mathrm{O}-\mathrm{H}}$ band, attributed to the allowed transition, should be more intense than the other band branch related with the forbidden transition. Therefore, based on these intuitive predictions, the spectral properties of the carboxylic acid dimers from the "b" group seem to be highly surprising, contradicting the interpretation of the spectra of systems belonging to the "a" and " $\mathrm{c}$ " groups. The particular electronic properties of the carboxylic acid molecules from the "c" group can anyway explain the extremely high integral intensity of the forbidden lower-frequency branch of the band and its strong temperature dependence.

In order to propose a reliable explanation of this paradox in our analysis, one should also recall the hydrogen bond IR spectra of other hydrogen bond dimeric systems, including spectra of hydrogen-bonded heterocycles. On comparison of the IR spectra of diverse crystalline systems containing cyclic hydrogen bond dimers as the structural units of their lattices, the following general conclusions can be made: most of centrosymmetric hydrogen bond dimers exhibit regular enough spectral properties characterizing their hydrogen bond spectra. Usually, the $\nu_{\mathrm{X}-\mathrm{H}}$ bands have the lowerfrequency (i.e., the "forbidden") branch of a lower intensity, even in their low-temperature spectra. However, in some rare cases, for example, 3-hydroxy-4-methyl-2(3H)-thiazolethione [36], 2-tiopyridone [37], and 2-pyridone [38], the $\nu_{\mathrm{O}-\mathrm{H}}$ and $\nu_{\mathrm{N}-\mathrm{H}}$ bands are characterized by an abnormal, that is, by a "reverse" intensity distribution patterns in their contours. In the latest cases, the lower-frequency branch of each band is more intense when compared with the higher-frequency band intensities. It fairly resembles the properties of the spectral properties at $77 \mathrm{~K}$ of carboxylic acid crystals of the "b" group. In the case of the dimeric spectra of the reverse intensity distribution patterns in the bands, for example, 3-hydroxy-4-methyl-2(3H)-thiazolethione [36] and 2-tiopirydone [37], this effect was ascribed previously to the influence of the extreme lengths of the $\mathrm{O}-\mathrm{H} \cdots \mathrm{S}$ and $\mathrm{N}-\mathrm{H} \cdot \cdots \mathrm{S}$ hydrogen bonds in the dimeric systems.

The recent considerations, aiming to explain these phenomena, were performed in terms of the dipole-dipole model of the vibrational exciton interactions involving the hydrogen bonds in the dimers. In the case of the interpretation of the spectra of 3-hydroxy-4-methyl-2(3H)-thiazolethione [36] and 2-tiopirydone [37], the hydrogen bond geometry
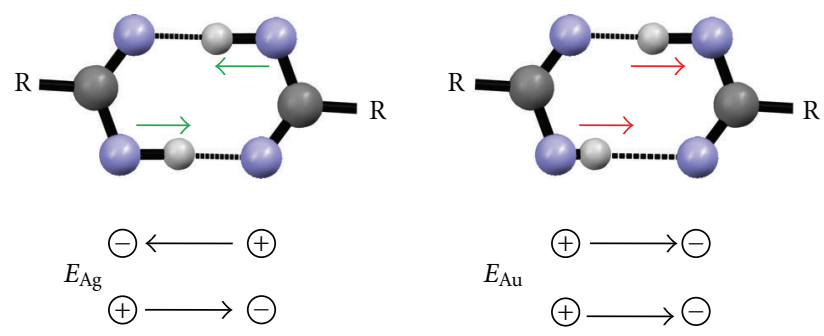

$E_{\mathrm{Au}}>E_{\mathrm{Ag}}$

FIGURE 12: The "side-to-side" (SS) exciton coupling involving the proton stretching vibrations in a cyclic centrosymmetric hydrogen bond dimer.

was considered to be responsible for the unusual spectral property of these dimers. However, this approach fails in the interpretation of the spectra of 2-pyridone cyclic dimers [38], in which the $\mathrm{N}-\mathrm{H} \cdots \mathrm{O}$ hydrogen bonds are considerably shorter when compared with the $\mathrm{N}-\mathrm{H} \cdots \mathrm{S}$ bond lengths in 2-thiopyridone cyclic dimers [37] and their spectra qualitatively fairly resemble the corresponding spectra of 2pyridone [38]. On the other hand, even among the hydrogen bond dimers of diverse molecular systems with the $\mathrm{N}-$ $\mathrm{H}$...S hydrogen bonds, for which the extreme spectral properties were found, a substantial diversification in the analyzed spectral properties has been found, despite the extremely long hydrogen bonds in these cases. The IR spectra of 2-mercaptobenzothiazole cyclic dimers [39] exhibit regular properties of the intensity distribution pattern in their $\nu_{\mathrm{N}-\mathrm{H}}$ band contours, similarly as the carboxylic acid dimers in the crystals of the groups "a" an "b," regardless of the extreme $\mathrm{N}-\mathrm{H} \cdots \mathrm{S}$ bond lengths, like these found in 2-thiopyridone dimers [37].

6.3. Spectra of Cyclic Dimers versus Spectra of Chain Hydrogen Bond Systems. It is surprising that spectra of cyclic hydrogen bond dimers in 3-hydroxy-4-methyl-2(3H)-thiazolethione [36], 2-thiopyridone [37], and 2-pyridone [38] crystals fairly resemble by their intensity distribution patterns of the $\nu_{\mathrm{N}-\mathrm{H}}$ bands the spectra of chain hydrogen bond systems in a particular group of molecular crystals. In the hydrogen bond spectra of pyrazole [40] and 4-thiopyridone [41] crystals, with hydrogen-bonded molecules forming infinite chains in their lattices, strong linear dichroic effects can be observed, which prove a considerable influence of the exciton interactions involving the adjacent hydrogen bonds in each chain. Figures 12 and 13 explain the source of the differences in the hydrogen bond dimers, the cyclic and the chain ones.

The analysis of this inter-hydrogen-bond coupling, in case of cyclic centrosymmetric dimers and in linear dimers, requires taking into consideration two situations of the vibrational transition moment directions for hydrogen bonds in the dimers. For cyclic dimers, the parallel mutual orientation of the dipole transition moments, the exciton interaction energy $E_{\mathrm{Au}}$ in the limits of the dipole-dipole model is of the positive sign. The vibrational transition corresponding to such arrangement of the vibration dipole moments is 

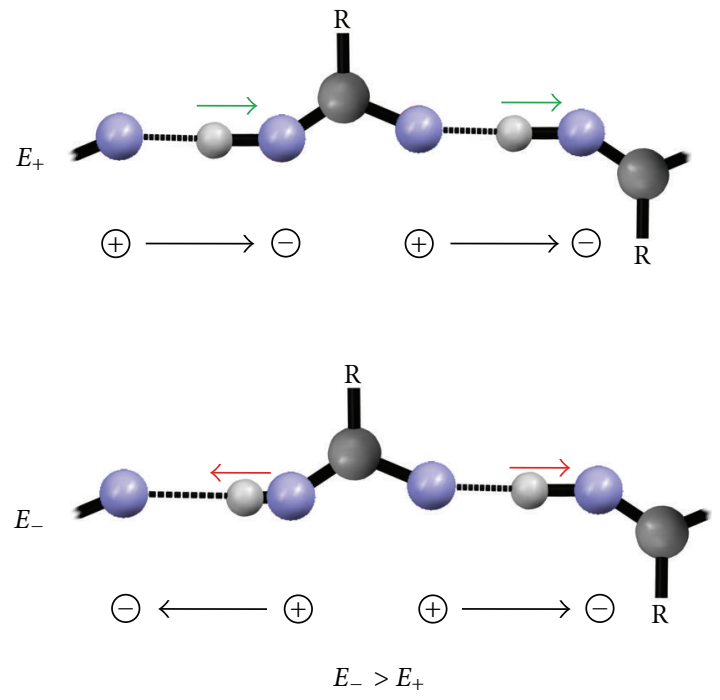

FIGURE 13: The "head-to-tail" (TH) exciton coupling involving the proton stretching vibrations in an infinite chain of associated hydrogen bonds.

responsible for generation of the intense, symmetry-allowed, shorter-wave branch of the dimeric spectra. In contrast, when the dipole transition moments are of the antiparallel arrangement (see Figure 12), the energy exciton interaction energy value $E_{\mathrm{Ag}}$ is negative, so the band generated by this situation is placed at the lower frequency and it corresponds to the symmetry-forbidden excitation of the totally symmetric proton vibrations. Such sequence of the spectral branches in the hydrogen bond stretching bands is typical for cyclic, centrosymmetric hydrogen bond dimers.

When the vibrating transition moment dipoles in a linear dimer, in the case of the totally symmetric proton vibrations, are oriented axially as "tail-to-head" (Figure 5), the sign of the exciton interaction energy value $E_{+}$is negative, so the intense branch corresponding to the symmetry-allowed transition is placed at the lower-frequency range. On the contrary, the forbidden by the symmetry rules spectral branch, situated at the higher frequency, is generated by the antiparallel orientation of the vibrating dipoles (see Figure 13). In this case, the exciton coupling energy $E_{-}$is of the positive sign. The sequence and the properties of the branches in the proton stretching vibration bands in the discussed case are reverse to those observed in the IR spectra of hydrogen bond cyclic dimeric systems.

Therefore, the following problem demands explanation: why do some individual cyclic hydrogen bond dimeric systems exhibit similar spectral properties to the corresponding properties of a particular group of crystals with chain structures of hydrogen-bonded associates (formic acid [31], pyrazole [40], and 4-thiopyridone [41] crystals). Undoubtedly, this property remains in a close connection with the $\pi$-electronic properties of the associating molecules. In the associated molecular systems, vibrational exciton couplings are of the "tail-to-head" (TH) type. They involve the adjacent hydrogen bonds within each individual chain in the lattice.
The electronic structure of molecules of this group is most probably the key factor governing these interhydrogen bond interactions.

Nevertheless, the majority of crystals with hydrogenbonded molecular chains in their lattices surprisingly exhibit the spectral properties similar to the analogous properties of cyclic hydrogen bond dimer spectra from the "a" and "c" groups (e.g., acetic acid [30], N-methylthioacetamide [42], or acetanilide [18] crystals). In the latest case the exciton interactions of the "side-to-side" (SS) type involve the closely spaced hydrogen bonds where each moiety belongs to a different chain. In molecules of this group, large $\pi$-electronic systems are absent. Only carbonyl or thiocarbonyl groups, each with a small $\pi$-electronic system, are present in these molecules.

From the above-presented data, it results that the way of realization of the vibrational exciton interactions in various hydrogen bond aggregates (cyclic dimers, infinite chains), affecting the $\nu_{\mathrm{X}-\mathrm{H}}$ and $\nu_{\mathrm{X}-\mathrm{H}}$ band fine structures, does not directly depend on the hydrogen bond system geometry. It is rather determined by the electronic structure of the associating molecules.

\section{Theoretical Approach Proposed}

The dipole-dipole interaction model, widely used for a simplified description of the exciton interactions between hydrogen bonds, seems to be nonadequate in the explanation of the wide diversity of the spectra of cyclic hydrogen bond dimers. There is some experimental data indicating that these couplings do not always occur as "through-space" and they are also widespread by the hydrogen bond electrons as well as by electrons of the molecular skeletons. Therefore, in terms of the theory of molecular vibrational excitons [43, 44], the exciton interaction integrals in some cases may also considerably strongly depend on the electronic coordinates. In advantageous circumstances, resulting from a proper electronic structure of the associating molecules, the proton stretching vibrations can induce electric current oscillating around a cyclic hydrogen bond dimer, or in the other case, oscillating along a hydrogen bond chain. However, only the totally symmetric proton vibrations are able to effectively induce the electric current in the ring or in the chain, while the nontotally symmetric vibrations are inactive in this mechanism, since currents induced in each individual hydrogen bond are annihilated in a dimer. The formalism of the model of the electric current generated by oscillating protons in cyclic hydrogen bond dimers was proposed by Nafie three decades ago [45].

In the scope of the considerations given above, it seems justified to treat formally a cyclic hydrogen bond dimer by the following two ways, taking into account the exciton interactions in the system.

(1) As a closed chain in which the adjacent hydrogen bonds are strongly exciton-coupled, similarly as in the chain associates in pyrazole [40] and 4-thiopyridone [41] crystals. This is the coupling of the TH type occurring around the molecular cycle. This 
way the coupling occurs via the easy-polarizable electrons on the $\pi$-orbitals. Therefore, the cyclic dimer spectrum is fairly similar to the spectrum of a chain system, with a low intensity of the higherfrequency band branch.

(2) As a pair of partially independent hydrogen bonds, which remains only "through-space" exciton coupled. It can be considered as a coupling of the SS type, without the generation of the ring electric current in the dimer. This behavior characterizes the associated molecular systems with no large $\pi$-electronic systems in their structures, where only small $\pi$-electronic systems are present in carbonyl and thiocarbonyl groups. In these circumstances, the dimeric spectra are of the standard form, with a low intensity of the lower-frequency $\nu_{\mathrm{X}-\mathrm{H}}$ band branch. For the quantitative description of the exciton interactions involving hydrogen bonds, influencing the dimer spectra, the dipole-dipole model is sufficiently adequate.

The $\nu_{\mathrm{X}-\mathrm{H}}$ band shapes in the two types of the dimer spectra are related one with the other by the approximate mirror reflection symmetry. In the case 1, the lower-intensity spectral branch appears in the higher-frequency range and is generated by the quasiforbidden vibrational transition in a dimer, occurring to the excited state of the totally symmetric proton stretching vibrations. In case 2 the lower intensity spectral branch appears in the lower-frequency range. It corresponds with the quasi-forbidden vibrational transition in a dimer. The above-presented spectral properties of diverse hydrogen bond cyclic dimers may allow explaining the thermal evolution effects in the hydrogen bond IR spectra of carboxylic acid crystals.

It seems that in order to explain the temperature effects in the IR spectra of cyclic hydrogen bond dimers the following hypothesis concerning the mechanisms of the spectra generation should be accepted: let us assume that two competing mechanisms of vibrational exciton interactions involving hydrogen bonds in cyclic dimers are simultaneously responsible for the formation of the $v_{\mathrm{X}-\mathrm{H}}$ band contour shapes. The contribution of each individual mechanism depends on the electronic structure of the associating molecules, on the electronic properties of the heavy atoms forming the hydrogen bridges as well as on temperature.

(A) The first mechanism depends on the "side-to-side" (SS)-type vibrational exciton coupling between the hydrogen bonds in cyclic dimers. In this case, the dimer hydrogen bonds interact one with the other as through-space via the van der Waals forces.

(B) The other mechanism assumes a "tail-to-head" (TH)type exciton coupling involving the hydrogen bonds in the dimers. These interactions occur around the cycles via electrons.

The " $\mathrm{B}$ " mechanism seems to be privileged in the case of the particular kind of associated molecules, in which hydrogen bonds couple with large $\pi$-electronic systems, for example, for aromatic carboxylic acid molecules. The "A" mechanism seems to dominate in the case of molecular systems with small $\pi$-electronic systems, for example, for aliphatic carboxylic acid molecules.

It seems obvious that for an individual hydrogen-bonded dimeric system the contribution of each mechanism is temperature dependent. For molecules with large $\pi$-electronic systems directly coupled with the hydrogen bonds, the "B" mechanism should be privileged at very low temperatures. Temperature growth, influencing the increase of atomic vibration amplitudes, should annihilate the electric current induced by the totally symmetric proton vibrations in the cycles. In these circumstances, the role of the "A" mechanism increases, namely, of the "through-space" vibrational exciton coupling between the hydrogen bonds in a dimer. This should, therefore, result in a particularly strong temperatureinduced evolution of the $v_{\mathrm{X}-\mathrm{H}}$ bands, especially in the case of the spectra of 2-thiopyridone [37] and 2-pyridone [38] type dimers. Even when the lower-frequency branch of the band is less intense when compared with the higher-frequency one, the temperature decrease till $77 \mathrm{~K}$ causes its considerable intensity growth, and in these circumstances the lowerfrequency branch becomes more intense than the higherfrequency band branch.

In the spectra of cyclic dimers, with only small $\pi$ electronic systems in the associating molecules, the temperature decrease usually does not cause a considerable intensity growth of the lower-frequency band branch. It still remains less intense when compared with the higher-frequency branch of the band. It means that, due to the molecular electronic properties of this group of dimers, the " $\mathrm{B}$ " mechanism cannot be activated effectively enough even at very low temperatures.

\section{Spectral Consequences of the Model for Carboxylic Acids}

From the above assumptions, it results the choice of the proper way of the model calculations of the $\nu_{\mathrm{X}-\mathrm{H}}$ and $\nu_{\mathrm{X}-\mathrm{D}}$ band contours in IR spectra of hydrogen bond dimers. In the limits of the proposed approach, a theoretical spectrum of the model system can be derived, formally treated as a superposition of two component spectra, where each individual spectrum corresponds with a different mechanism of the exciton interactions, SS (A) and TH (B), involving the dimer hydrogen bonds. In terms of the "strong-coupling" theory [6-8], in each exciton interaction mechanism case, the $\nu_{\mathrm{X}-\mathrm{H}}$ band in the dimeric spectrum is a superposition of two component bands, "Plus" and "Minus," each of a different origin.

The "Plus" band is generated by the dipole allowed transition to the excited state of the nontotally symmetric proton stretching vibrations in a centrosymmetric dimer, belonging to the $\mathrm{Au}$ representation. On the other hand, the "Minus" band is connected with the symmetry forbidden transition to the Ag-symmetry state of the totally symmetric proton vibrations in the dimers, activated by a vibronic mechanism [35]. In the case when the mechanism " $\mathrm{A}$ " exclusively decides about the dimer spectra generation mechanism, the "Minus" band appears in the lower "B" mechanism frequency range in 
relation to the "Plus" band location. In the other case, when the " $\mathrm{B}$ " mechanism governs the dimer spectra generation, the two component bands appear in the reverse sequence than in the case "A." It means that in the case of the "B" mechanism governing the spectra generation, the "Minus" band representing the forbidden transition appears in the higher-frequency range than the "Plus" band connected with the allowed transition.

\section{Model Calculations of the Band Contours}

In the two cases, $\mathrm{A}$ and $\mathrm{B}$, model calculations, aiming at reconstituting of the "residual" $\nu_{\mathrm{O}-\mathrm{H}}$ and $\nu_{\mathrm{O}-\mathrm{D}}$ band shapes, were performed within the limits of the "strong-coupling" theory, for a model centrosymmetric $\mathrm{O}-\mathrm{H} \cdots \mathrm{O}$ hydrogen bond dimeric system. $[6-8,46]$. We assumed that the main $\nu_{\mathrm{O}-\mathrm{H}}$ and $\nu_{\mathrm{O}-\mathrm{D}}$ band shaping mechanism involved strongly anharmonically coupled the high-frequency proton (or deuteron) stretching vibrations and the low-frequency $\mathrm{O} \cdots \mathrm{O}$ hydrogen bridge stretching vibrational motions. Calculation of the hydrogen bond system IR spectra in terms of the "strong-coupling" model allows to obtain results fairly comparable with the results of the spectra calculation performed using the "relaxation" theory $[9,10,47-49]$.

According to the formalism of the "strong-coupling" theory $[6-8,46]$, the $\nu_{\mathrm{O}-\mathrm{H}}$ band shape of a dimer depends on the following system of dimensionless coupling parameters: (i) on the distortion parameter, " $b_{H}$ ", and (ii) on the resonance interaction parameters, " $C_{O}$ " and " $C_{1}$ ". The " $b_{H}$ " parameter describes the change in the equilibrium geometry for the low-energy hydrogen bond stretching vibrations, accompanying the excitation of the high-frequency proton stretching vibrations $\nu_{\mathrm{O}-\mathrm{H}}$. The " $\mathrm{C}_{O}$ " and " $C_{1}$ " parameters are responsible for the exciton interactions between the hydrogen bonds in a dimer. They denote the subsequent expansion coefficients in the series on developing the resonance interaction integral " $\mathrm{C}$ " with respect to the normal coordinates of the $\nu_{\mathrm{O}} \ldots \mathrm{O}$ low-frequency stretching vibrations of the hydrogen bond. This is in accordance with the formula:

$$
C=C_{O}+C_{1} Q_{1},
$$

where $Q_{1}$ represents the totally symmetric normal coordinate for the low-frequency hydrogen bridge stretching vibrations in the dimer. This parameter system is closely related to the intensity distribution in the dimeric $v_{\mathrm{N}-\mathrm{H}}$ band. The " $b_{H}$ " and " $C_{1}$ " parameters are directly related to the dimeric $\nu_{\mathrm{N}-\mathrm{H}}$ component bandwidth. The " $C_{O}$ " parameter defines the splitting of the component bands of the dimeric spectrum corresponding to the excitation of the proton vibrational motions of different symmetries, $\mathrm{Ag}$ and $\mathrm{Au}$. In its simplest, original version, the "strong-coupling" model predicts reduction of the distortion parameter value for the deuterium bond systems according to the relation:

$$
b_{H}=\sqrt{2} b_{D} .
$$

For the " $C_{O}$ " and " $C_{1}$ " resonance interaction parameters, the theory predicts the isotopic effect expressed by the 1.0 to $\sqrt{2}$-fold reduction of the parameter values for $D$-bonded dimeric systems.

As the consequence of the "strong-coupling" model, the $\nu_{\mathrm{O}-\mathrm{H}}$ and $\nu_{\mathrm{O}-\mathrm{D}}$ band contour fine structures were treated as a superposition of two component bands. They correspond to the excitation of the two kinds of proton stretching vibrations, each exhibiting a different symmetry. In the case of the A exciton coupling mechanism and for the $C_{i}$ point symmetry group of the model dimer, the excitation of the $\mathrm{Ag}$ vibrations in the dimer generates the lower-frequency transition branch of the $\nu_{\mathrm{O}-\mathrm{H}}$ band when the Au vibrations are responsible for the higher-frequency band branch. In the case of the B mechanism, the component subbands appear in reverse sequence.

Here, we consider an identical anharmonic coupling parameter system for the two individual mechanism cases $\mathrm{A}$ and $\mathrm{B}$ although diversification of the coupling parameter value systems seems to be better justified. We assume the contribution of each mechanism as governed by a Boltzmanntype relation. In addition, for the statistical weight parameters of each individual mechanism, $P_{\mathrm{A}}(T)$ and $P_{\mathrm{B}}(T)$, one must distinguish which state is dominant, that is, when the SS (A) state is of the lower energy and the TH (B) state is of a higher energy value and vice versa. In order to reproduce the temperature dependence of experimental spectra particularly for its width and the position of its first moment, we used for the $P_{\mathrm{A}}^{\mathrm{AB}}(T)$ exponential temperature dependence according to

$$
P_{\mathrm{A}}^{\mathrm{AB}}(T)=1-\exp \left(-\frac{\alpha^{\mathrm{AB}}}{k_{\mathrm{B}} T}\right)
$$

where is $\alpha^{\mathrm{AB}}$ the activation energy parameter when the SS state is dominant and $\mathrm{k}_{\mathrm{B}}$ is the constant of Boltzmann. In such circumstance, $P_{\mathrm{B}}^{\mathrm{AB}}(T)$ takes the following expression:

$$
P_{\mathrm{B}}^{\mathrm{AB}}(T)=\exp \left(-\frac{\alpha^{\mathrm{AB}}}{k_{\mathrm{B}} T}\right),
$$

It is interesting to note that, in the case of A, for very low temperatures, the statistical weight $P_{\mathrm{A}}^{\mathrm{AB}}(T)$ parameter is close to 1.0 and $P_{\mathrm{B}}^{\mathrm{AB}}(T)$ is almost equal 0.0. In these circumstances, the SS-type interaction is the basic type of the exciton coupling involving the dimer hydrogen bonds. For high temperatures, the $P_{\mathrm{B}}^{\mathrm{AB}}(T)$ parameter values are different from 0.0 and they are intermediate between 0.0 and 1.0 (rather closer to 0.5 ) and $P_{\mathrm{A}}^{\mathrm{AB}}(T)$ approaches 0.5. When the temperature increases, $P_{\mathrm{B}}^{\mathrm{AB}}(T)$ also increases. It means that, the $\mathrm{TH}$ coupling, occurring via the electric current in the ring is activated in higher temperatures in a magnitude depending of the energy gap between these two states of the vibrationally excited dimer. From our experimental estimations, the energy gap for some dimeric system cases is relatively large and in another cases it may be relatively low.

In the case $\mathrm{B}$, where the $\mathrm{TH}$ state is of a lower energy value, we assume the same formula but the energy barrier $\alpha^{\mathrm{BA}}$ height is relatively low. In such a circumstance, 
the statistical weight parameters, $P_{\mathrm{A}}(T)$ and $P_{\mathrm{B}}(T)$, may be written as follows:

$$
\begin{gathered}
P_{\mathrm{A}}^{\mathrm{BA}}(T)=\exp \left(-\frac{\alpha^{\mathrm{BA}}}{k_{\mathrm{B}} T}\right), \\
P_{\mathrm{B}}^{\mathrm{BA}}(T)=1-\exp \left(-\frac{\alpha^{\mathrm{BA}}}{k_{\mathrm{B}} T}\right) .
\end{gathered}
$$

As we can see, for very low temperatures, $P_{\mathrm{A}}^{\mathrm{AB}}(T)$ may be practically equal to 1.0. For this kind of dimeric systems, the TH-type exciton coupling is the basic natural way in which the inter-hydrogen bond interactions occur. The growth in temperature annihilates this way of the coupling, due to the vanishing of the electronic current induced in the cycles, accompanied by large-amplitude thermal motions of atoms in the dimers. For high temperatures, $P_{\mathrm{A}}^{\mathrm{AB}}(T)$ decreases and becomes of an intermediate value between 0.0 and 1.0 (rather closer to 0.5 ), while the statistical weight $P_{\mathrm{A}}^{\mathrm{AB}}(T)$ grows declining from 0.0 up to 0.5 . The energy gap between the two states in some molecular cases is usually relatively large, and in other cases it may be relatively small. It depends of the electronic properties of the associating molecules forming the dimers. From our experimental data, it can be concluded that the cases A and B represent the extreme cases of the interhydrogen bond coupling in cyclic hydrogen bond dimers. There are also many systems exhibiting an intermediate behavior. For a relatively small magnitude of the absolute values of the energy barrier height, the two cases $\mathrm{A}$ and $\mathrm{B}$ are practically nondistinguishable.

The theoretical spectra reconstituting the $\nu_{\mathrm{O}-\mathrm{H}}$ band contours measured at the two different temperatures, $293 \mathrm{~K}$ and $77 \mathrm{~K}$, were calculated in terms of the two different individual coupling mechanisms, SS and $\mathrm{TH}$, which generate the two component bands, "plus" and "minus" in a different sequence. The following coupling parameter values, identical in both molecular system cases, were used.

For the 2-furanacetic acid crystal spectra: $\mathrm{b}_{\mathrm{H}}=1.6, C_{0}=$ $1.5, C_{1}=-0.2, F^{+}=1.0, F^{-}=0.2, \Omega_{\mathrm{O}} \cdots \mathrm{O}=100 \mathrm{~cm}^{-1}$, and we used the same parameter system for calculation of the 2furanacrylic acid crystal spectra: $b_{H}=1.6, C_{0}=1.5, C_{1}=-0.2$, $F^{+}=1.0, F^{-}=0.2, \Omega_{\mathrm{O}} \ldots \mathrm{O}=100 \mathrm{~cm}^{-1}$.

The $F^{+}$and $F^{-}$symbols denote the statistical weight parameters for the "plus" and "minus" theoretically derived subspectra contributing at the band formation.

The coupling parameter values used for calculation of the $\nu_{\mathrm{O}-\mathrm{D}}$ band contour shapes were as follows.

For 2-furanacetic acid crystal, spectrum $b_{D}=0.7, C_{0}=$ $0.7, C_{1}=-0.1, F^{+}=1.0, F^{-}=0.2, \Omega_{\mathrm{O}} \ldots \mathrm{O}=100 \mathrm{~cm}^{-1}$ and for 2-furanacrylic acid crystal spectrum: $b_{H}=0.7, C_{0}=0.7, C_{1}=$ $-0.1, F^{+}=1.0, F^{-}=0.2, \Omega_{\mathrm{O}} \cdots \mathrm{O}=100 \mathrm{~cm}^{-1}$.

For the 2-furanacetic acid crystal spectra the statistical weight parameter ratio, $P_{\mathrm{A}}(T): P_{\mathrm{B}}(T)$, for the SS and TH mechanisms was estimated as equal to $1.0: 0.0$ in the case of the room temperature spectrum reconstitution. For the lowtemperature spectrum case, this parameter ratio value is very similar and equal to $1.0: 0.0$. Among various parameter ratio values for the SS and TH mechanisms contributing in the band generation, this parameter ratio value allowed for the

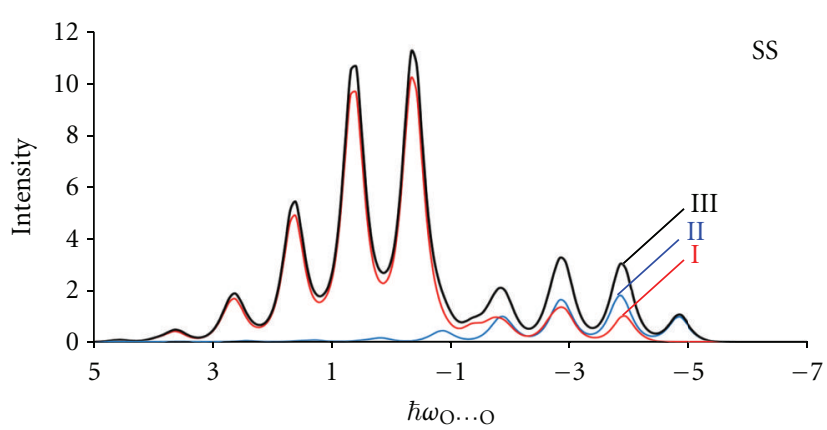

(a)

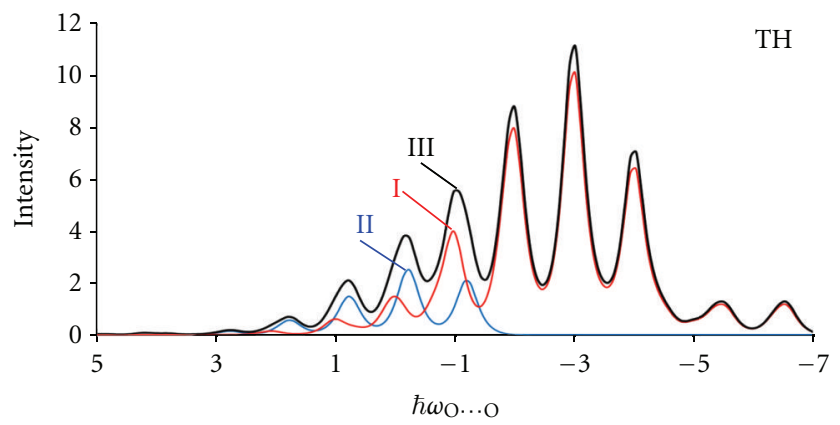

(b)

FIGURE 14: The theoretically derived $\nu_{\mathrm{O}-\mathrm{H}}$ band contours calculated in terms of the "strong-coupling" theory in the limits of the two different vibrational exciton coupling mechanisms involving the cyclic dimer hydrogen bonds, that is, "side-to-side" (SS) and "tail-tohead" (TH). (a) The SS coupling mechanism. (b) The TH coupling mechanism. (I) The "minus" band. (II) The "plus" band (III) Superposition of the I and II spectra, each taken with its appropriate individual statistical weight parameter, $F^{-}$and $F^{+}$. In both mechanism cases, the same coupling parameter value system was used for calculations: $b_{H}=1.4, C_{0}=1.5, C_{1}=-0.2, \mathrm{~F}^{+}=1.0$, $F^{-}=0.2, \Omega_{\mathrm{O}} \ldots \mathrm{O}=100 \mathrm{~cm}^{-1}$. The transition frequencies are in the $\omega_{\mathrm{O}} \ldots \mathrm{O}$ vibrational quantum units, and the transition frequencies are expressed with respect to the gravity center of the hypothetical spectrum of a monomeric hydrogen bond in the cyclic hydrogen bond dimer. Transition intensities are in arbitrary units.

most adequate reproduction of the temperature effect in the crystal spectra.

For the 2-furanacrylic acid crystal spectra, the statistical weight parameter ratio, $P_{\mathrm{A}}(T): P_{\mathrm{B}}(T)$, for the SS and TH mechanisms were estimated as equal to $0.35: 0.65$ in the case of the room temperature spectrum reconstitution. For the low-temperature spectrum case, this parameter ratio value is equal to $0.55: 0.45$.

In Figures 14 and 15, we present the theoretical $\nu_{\mathrm{O}-\mathrm{H}}$ and $\nu_{\mathrm{O}-\mathrm{D}}$ band contours calculated in terms of the two individual mechanisms of the vibrational exciton interactions involving the dimer hydrogen bonds, SS and TH.

In Figures 16 and 17, the evolution of the $\nu_{\mathrm{O}-\mathrm{H}}$ and $\nu_{\mathrm{O}-\mathrm{D}}$ band contour shapes accompanying the variation in the relative contribution of the SS and TH coupling mechanisms in generation of a dimeric spectra is shown. Similar band 


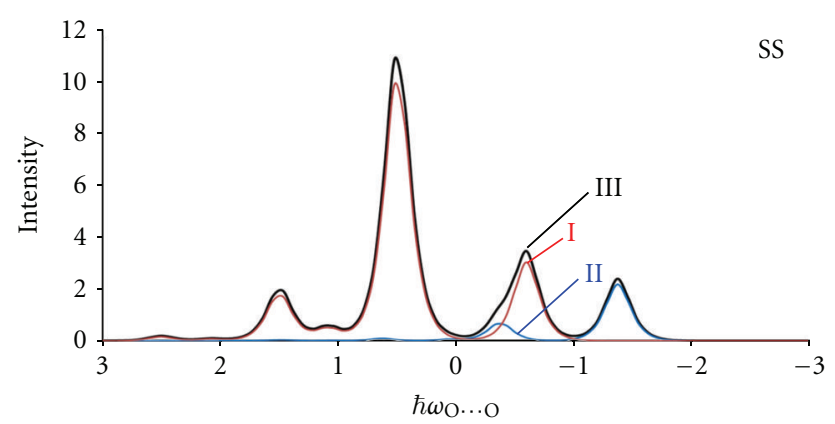

(a)

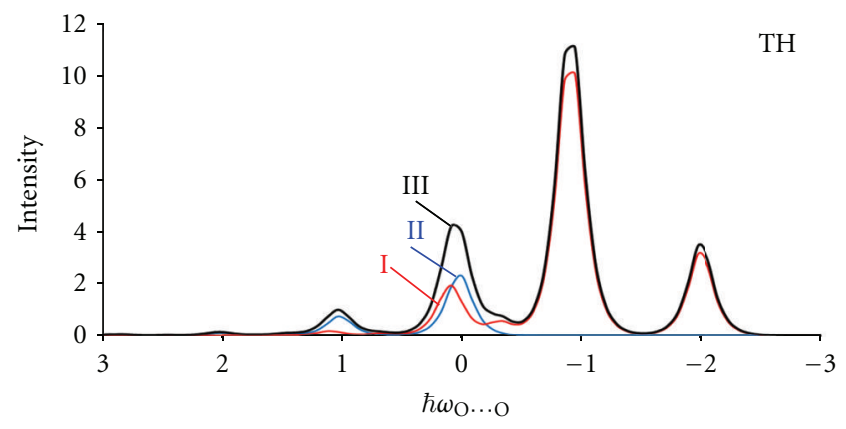

(b)

FIGURE 15: The theoretically derived $\nu_{\mathrm{O}-\mathrm{D}}$ band contours calculated in terms of the "strong-coupling" theory in the limits of the two different vibrational exciton coupling mechanisms involving the cyclic dimer hydrogen bonds, that is, "side-to-side" (SS) and "tail-tohead" (TH). (a) The SS coupling mechanism. (b) The TH coupling mechanism. (I) The "minus" band. (II) The "plus" band. (III) Superposition of the spectra I and II, each taken with its appropriate individual statistical weight parameter, $F^{-}$and $F^{+}$. In both mechanism cases, the same coupling parameter value system was used for calculations: $b_{H}=0.7, C_{0}=0.7, C_{1}=-0.2, F^{+}=1.0$, $F^{-}=0.2, \Omega_{\mathrm{O}} \ldots \mathrm{O}=100 \mathrm{~cm}^{-1}$. The transition frequencies are in the $\omega_{\mathrm{O}} \cdots \mathrm{O}$ vibrational quantum units, and the transition frequencies are expressed with respect to the gravity center of the hypothetical spectrum of a monomeric hydrogen bond in the cyclic deuterium bond dimer. Transition intensities are in arbitrary units.

shape evolution accompanies temperature changes during the spectral experiments.

From the comparison of the corresponding calculated and experimental spectra, it results that the intensity distribution patterns and the temperature effects in the spectra of the two different crystalline systems have been at least semiquantitatively reproduced via the model calculations.

\section{Spectra of 2-Furanacetic and 2-Furanacrylic Acid Crystals}

On comparing the IR spectra of the hydrogen bond for the two crystalline systems, essential differences analyzed crystalline spectra othe $\nu_{\mathrm{O}-\mathrm{H}}$ and $\nu_{\mathrm{O}-\mathrm{D}}$ bands. In the case of 2furanacetic acid spectra, the fine structure pattern of each band, $v_{\mathrm{O}-\mathrm{H}}$ and $\nu_{\mathrm{O}-\mathrm{D}}$, is relatively simple. Each band consists of a low number of well-separated spectral lines. In the

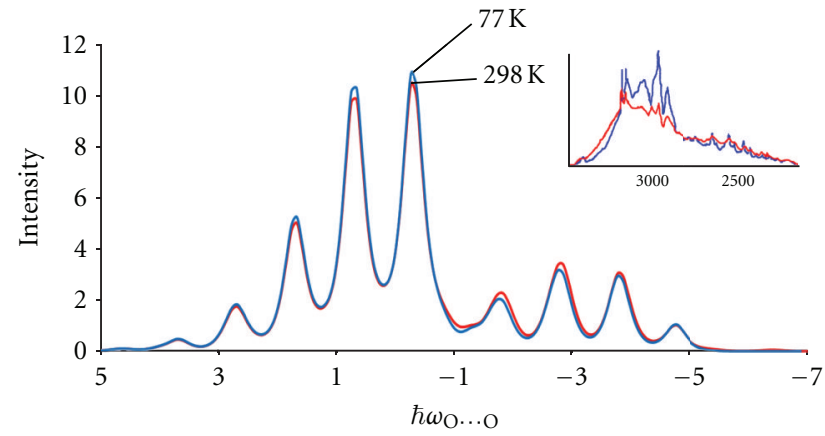

(a)

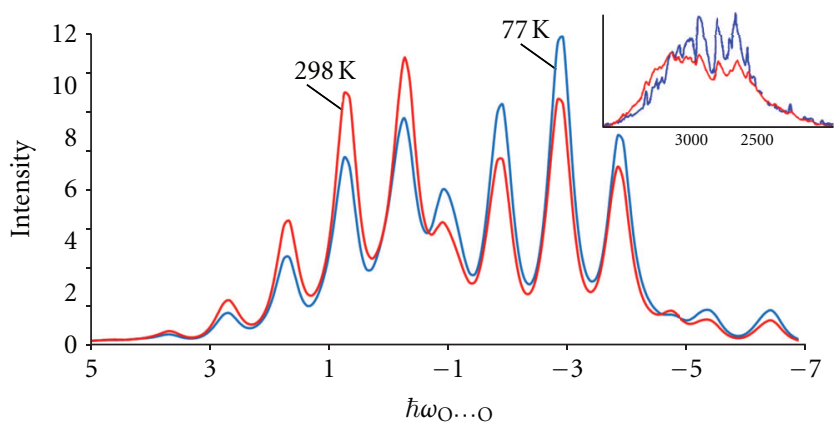

(b)

Figure 16: Temperature-induced evolution of the $\nu_{\mathrm{O}-\mathrm{H}}$ band contour shapes accompanying the variation in the contribution rate of the two different exciton coupling mechanisms, that is, "sideto-side" (SS) and "tail-to-head" (TH). Numerical reproduction of the temperature effect in the spectra of hydrogen-bonded (a) 2furanacetic acid crystal (b) 2-furanacrylic acid crystal. The relative contribution ratio of the SS and TH mechanisms in the $v_{\mathrm{O}-\mathrm{H}}$ band generation is, for 2-furanacetic acid crystal: $0.95: 0.05$ at $293 \mathrm{~K}$ and $0.95: 0.05$ at $77 \mathrm{~K}$ and for 2-furanacrylic acid crystal: $0.65: 0.35$ at $293 \mathrm{~K}$ and $0.40: 0.60$ at $77 \mathrm{~K}$. The experimental spectra are shown in inset.

spectra of 2-furanacrylic acid, each considered band is composed of a noticeably larger number of lines (ca. 2 times larger). It seems to prove a more complex mechanism of the spectra generation in the case of 2-furanacrylic acid in relation to the mechanism governing the spectra generation of 2-furanacetic acid.

The analyzed crystalline spectra of 2-furanacetic acid seem to fully belong to the case A. On the other hand, the crystalline spectra of 2-furanacrylic acid seem to satisfy the demands of the case B. The analyzed difference in the spectral properties of arylacetic acid dimers and the arylacrylic acid dimers most probably results from the influences exerted on to the hydrogen bond dimers, present in the $(\mathrm{COOH})_{2}$ cycles, by the aromatic rings. The direct contact between the furan rings with carboxyl groups (arylcarboxylic, furanacrylic, and thiopheneacrylic acids) most likely influences the electric charge density in the $(\mathrm{COOH})_{2}$ cycles. This in turn strengthens the vibronic mechanism of the electronic current generation in the hydrogen bond cycles [45]. Separation of the carboxyl groups from aromatic rings by methylene 


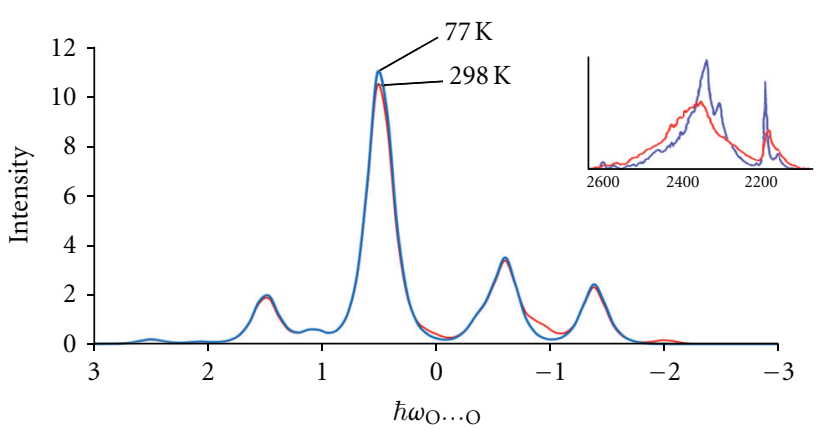

(a)

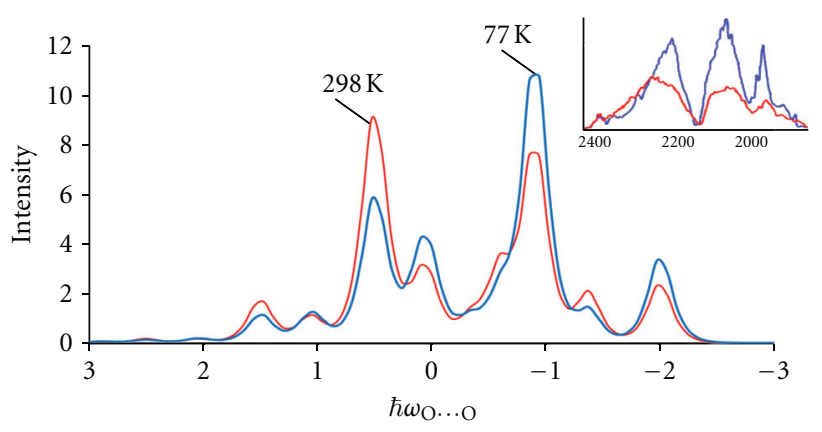

(b)

FIgURE 17: Temperature-induced evolution of the $\nu_{\mathrm{O}-\mathrm{D}}$ bandcontour shapes accompanying the variation in the contribution rate of the two different exciton coupling mechanisms, that is, "sideto-side" (SS) and "tail-to-head" (TH). Numerical reproduction of the temperature effect in the spectra of deuterium-bonded: (a) 2furanacetic acid crystal (b) 2-furanacrylic acid crystal.The relative contribution ratio of the SS and TH mechanism in the $\nu_{\mathrm{O}-\mathrm{D}}$ band generation is, for 2-furanacetic acid crystal: $0.95: 0.05$ at $293 \mathrm{~K}$ and $0.95: 0.05$ at $77 \mathrm{~K}$ and for 2-furanacrylic acid crystal: $0.65: 0.35$ at $293 \mathrm{~K}$ and $0.40: 0.60$ at $77 \mathrm{~K}$. The experimental spectra are shown in inset.

groups (arylacetic acids, furanacetic acids, and thiopheneacetic acids) effectively weakens the vibronic coupling mechanism. Therefore, these latter systems belong to the A case.

The analyzed spectral properties of the two different crystalline systems, 2-furanacetic acid and 2-furanacrylic acid, are in a good agreement with the described above vibrational exciton interaction mechanisms of the spectra generation for cyclic hydrogen bond dimer. This remains in a close relation to the electronic properties of the two carboxylic acid molecules. For 2-furanacetic acid dimers, the exciton interactions involving the dimer hydrogen bonds of a SStype is only weakly temperature dependent. In the case of 2furanacrylic acid dimers, due to their electronic structure, the interhydrogen bond exciton coupling mechanism changes its character along with the changes in temperature. At very low temperatures, the TH-type interactions, transferred in the $(\mathrm{COOH})_{2}$ cycles via electrons are dominating. When temperature increases, this mechanism becomes less privileged as being annihilated by the hydrogen-bond atom thermal vibrational motions. It is replaced by the other mechanism depending of the SS-type interactions. Each individual mechanism generates its own spectrum characterized by its unique intensity distribution pattern. Therefore, the $\nu_{\mathrm{O}-\mathrm{H}}$ and $\nu_{\mathrm{O}-\mathrm{D}}$ bands in the spectra of 2-furanacrylic acid crystals exhibit more complex fine structure patterns, since they are superposition of two different spectra, where each component spectrum is of a different origin. Each component spectrum contributing to the $\nu_{\mathrm{O}-\mathrm{H}}$ and $\nu_{\mathrm{O}-\mathrm{D}}$ band formation, with its statistical weight parameter depended of temperature, corresponds with another exciton interaction mechanism in the cyclic hydrogen bond dimers in the lattice.

Spectra of 2-thiopheneacrylic acid crystals [50] exhibit qualitatively fairly similar properties as the spectra of 2furanacrylic acid crystals. Their $\nu_{\mathrm{O}-\mathrm{H}}$ and $\nu_{\mathrm{O}-\mathrm{D}}$ bands also demonstrate complex and dense fine structure patterns. They also show very similar temperature effects when compared with the corresponding spectra of 2-furanacrylic acid crystals.

In turn, the spectra of 2-thiopheneacetic acid crystals [50] exhibit qualitatively very similar properties as the spectra of 2-furanacetic acid crystals. Their $\nu_{\mathrm{O}-\mathrm{H}}$ and $\nu_{\mathrm{O}-\mathrm{D}}$ bands also exhibit relatively simple fine structure patterns. They also demonstrate fairly similar temperature effects when compared with the corresponding spectra of 2-furanacetic acid crystals.

From the comparison of the spectra of the two different groups of carboxylic acid crystals, it results that the electronic structure of the associating molecules is the main factor determining the crystal spectral properties in IR, differentiating the spectral properties of the two groups of hydrogenbonded systems. Namely, the temperature effects registered in IR spectra of the hydrogen bond in carboxylic acid crystals remain in a close connection with the electronic spectra of the associating molecules forming cyclic hydrogen-bonded dimers in the lattices.

\section{The Problem of the Vibrational Selection Breaking in IR Spectra of Centrosymmetric Hydrogen Bond Dimers}

The mechanism proposed in this paper for understanding the sources of temperature effects in the IR spectra of cyclic centrosymmetric hydrogen bond dimers explains the generation of the lower-frequency $\nu_{\mathrm{O}-\mathrm{H}}$ and $\nu_{\mathrm{O}-\mathrm{D}}$ band branches of extremely high intensities in IR spectra of carboxylic acid crystals. However, at this stage, the relation with the formerly published vibronic mechanism of the vibrational rule selection breaking in the IR spectra of centrosymmetric hydrogen bond dimers [35] ought to be discussed since both mechanisms can generate and also explain qualitatively fairly similar spectral effects.

The vibronic mechanism was originally elaborated in the past for the understanding of the fine structure patterns of the published earlier IR spectra of the cyclic, centrosymmetric $\mathrm{N}-\mathrm{H}$. . S bond dimers formed by 2-thiopyridone and 2-mercaptobenzothiazole molecules as well as extremely nonregular $\mathrm{H} / \mathrm{D}$ isotopic effects in the spectra [37, 39, 51]. The isotopic effects were expressed by the unusually narrow $\nu_{\mathrm{N}-\mathrm{D}}$ bands in correspondence to the very wide $\nu_{\mathrm{N}-\mathrm{H}}$ bands characterized by complex fine structure patterns. In terms of the vibronic model, these effects were 
explained by the disappearance of the intensity of the lower-frequency branch of the $v_{\mathrm{N}-\mathrm{D}}$ bands attributed to the $\mathrm{N}-\mathrm{D}$ bond totally symmetric stretching vibrations in the dimers, due to the weakening of the forbidden transition promotion mechanism [35]. In the case of the $\nu_{\mathrm{N}-\mathrm{H}}$ bands, the promotion mechanism was effective enough generating the forbidden transition spectral branch of noticeably high intensity. Nevertheless, this branch appeared to be less intense when compared with the allowed transition, higherfrequency branch of the $\nu_{\mathrm{N}-\mathrm{H}}$ band. The vibronic model ascribed these effects to the difference in the proton and deuteron vibration anharmonicity and to the extremely high polarizability of the $\mathrm{N}-\mathrm{H} \cdots \mathrm{S}$ hydrogen bonds in 2thiopyridone and 2-mercaptobenzothiazole dimers. These factors were considered as responsible for the magnitude of the vibrational selection rule breaking effects in the dimeric IR spectra [35].

The IR spectra of carboxylic acid crystals with cyclic dimers in their lattices considerably differ by the analogous $\mathrm{H} / \mathrm{D}$ isotopic effects from the spectra of the $\mathrm{N}-\mathrm{H} \cdots \mathrm{S}$ bonded dimers [22-27, 37, 39]. In the case of carboxylic acid crystals practically no impact of the isotopic substitution onto the relative intensity of the lower-frequency band branch intensities of the $\nu_{\mathrm{O}-\mathrm{H}}$ and $\nu_{\mathrm{O}-\mathrm{D}}$ bands in relation to the corresponding higher-frequency band branch intensities. can be noticed. Also the incidentally observed very high intensities of the forbidden transition bands distinguish these IR spectra of carboxylic acid crystals. This proves that the spectra generation mechanism for the carboxylic acid dimers in the crystals essentially differs from the vibronic selection rule breaking mechanism [35].

The following question arises in the scope of our latest estimations: should the vibronic mechanism be definitively rejected as inadequate in the description of the IR spectral properties of centrosymmetric hydrogen bond dimers, especially carboxylic acid dimers in the solid state?

From our hitherto studies of IR spectra of hydrogenbonded molecular crystals, it results that the two different mechanisms forming the band structures act parallel, each with its individual statistical weight, depending of the electronic properties of the molecular systems forming the dimers. In the case of cyclic dimeric $\mathrm{N}-\mathrm{H} \cdots \mathrm{S}$ bonded molecular systems, the vibronic mechanism appeared to be relatively very sufficient, leading to the appearance of intense forbidden transition $\nu_{\mathrm{N}-\mathrm{H}}$ band branches. On the other hand, the $\nu_{\mathrm{N}-\mathrm{D}}$ bands are extremely narrow as practically devoid of the forbidden band branch [37, 39]. The vibronic mechanism is also effective in the generation of IR spectra of crystals with infinite open chains of hydrogen bonded molecules, for example, $\mathrm{N}$-methylthioacetamide [42] or Nphenylacrylamide [52] crystals. Also the H/D isotopic effects in their spectra are fairly similar to the analogous isotopic effects in the corresponding spectra of the $\mathrm{N}-\mathrm{H} \cdots$. S bonded cyclic dimers. In these chain structures, centrosymmetric hydrogen bond dimeric systems are composed of hydrogen bonds, where each moiety belongs to another chain of associated molecules penetrating a unit cell. Most probably, the chain structure of the molecular associates, which excludes the possibility of the induction to circulating electric currents in such dimers, as well as the polarization properties of these hydrogen bonds, is responsible for the existence of the vibronic mechanism [35] in the pure form, influencing the band contour formation.

For the carboxylic acid dimer spectra, the mechanism proposed in this work is dominant regardless of the electronic structure of the substituent atomic groups linked to the carboxyl groups in the molecules. On the basis of the "state-of-art" in the spectral studies of the hydrogen bond systems in molecular crystals, the H/D isotopic effects in the spectra seem to be the main criterion for distinguishing these two individual mechanisms. However, this problem demands further intensive studies in the future.

\section{Conclusions}

In this paper, we report experimental and theoretical study of IR spectra of 2-furanacetic acid and of 2-furanacrylic acid crystals measured at $293 \mathrm{~K}$ and $77 \mathrm{~K}$ in the $\nu_{\mathrm{O}-\mathrm{H}}$ and $\nu_{\mathrm{O}-\mathrm{D}}$ band frequency ranges. The corresponding spectra of the two individual systems strongly differ. Indeed, in the case of 2-furanacetic acid spectra, the fine structure pattern of each band, $\nu_{\mathrm{O}-\mathrm{H}}$ and $\nu_{\mathrm{O}-\mathrm{D}}$, is relatively simple. Each band consists of a low number of well-separated spectral lines. In the spectra of 2-furanacrylic acid, each considered band is composed of a noticeably larger number of lines. In addition, the temperature effect characterizing the bands is not the same for the two compounds. The results presented in this paper for 2-furanacetic acid and 2-furanacrylic acid allow for the following observations and conclusions.

(1) The crystal IR spectral properties remain in a close relation with the electronic structure of the two different molecular systems. The vibronic coupling mechanism involving the hydrogen bond protons and the electrons on the $\pi$-electronic systems in the molecules determines the way in which the vibrational exciton coupling between the hydrogen bonds in the carboxylic acid dimers occurs.

(2) The analyzed spectral properties of the two different crystalline systems, 2-furanacetic acid and 2furanacrylic acid, are in a good agreement with the vibrational exciton interaction mechanisms of the spectra generation for cyclic hydrogen bond dimer.

(3) For 2-furanacetic acid dimers, the exciton interactions involving the dimer hydrogen bonds of the SS type are only weakly temperature dependent. A weak "through-space" coupling in 2-furanacetic acid dimers of a van der Waals type is responsible for the SS-type coupling.

(4) In the case of 2-furanacrylic acid dimers, due to their electronic molecular structure, the interhydrogen bond exciton coupling mechanism strongly changes its character along with the changes in temperature. Strong coupling in 2-furanacrylic acid dimers prefers a TH-type Davydov coupling widespread by the $\pi$ electrons. At very low temperatures, the TH-type interactions, transferred in the $(\mathrm{COOH})_{2}$ cycles via 
electrons are dominating. This mechanism becomes less privileged at higher temperature as annihilated by the hydrogen-bond atom thermal vibrational motions.

(5) Each individual mechanism, that is, the TH and SS, generates its own spectrum characterized by its unique individual intensity distribution pattern. As we can see, the $v_{\mathrm{O}-\mathrm{H}}$ and $\nu_{\mathrm{O}-\mathrm{D}}$ bands in the spectra of 2-furanacrylic acid crystals exhibit more complex fine structure patterns, since they are superposition of two different spectra, where each component spectrum is of a different origin. Each component spectrum contributing to the $\nu_{\mathrm{O}-\mathrm{H}}$ and $\nu_{\mathrm{O}-\mathrm{D}}$ bands formation, with its temperature-dependent statistical weight, corresponds with the different exciton interaction mechanism, TH or SS, acting in the cyclic hydrogen bond dimers in the lattice. This explains the observed difference in the temperature-induced evolution of the compared spectra.

\section{References}

[1] C. Pimentel and A. L. McClellan, The Hydrogen Bond, W. H. Freeman and Co, San Francisco, Calif, USA, 1960.

[2] P. Schuster, G. Zundel, and C. Sandorfy, The Hydrogen Bond, vol. 1-3, North-Holland, Amsterdam, The Netherlands, 1976.

[3] G. L. Hofacker, Y. Marechal, and M. A. Ratner, "The dynamical aspects of hydrogen bonds," in In The Hydrogen Bond, Recent Developments in Theory and Experiment, W. P. Schuster, G. Zundel, and C. Sandorfy, Eds., vol. 1, p. 295, North-Holland, Amsterdam, The Netherlands, 1976.

[4] P. Schuster and W. Mikenda, Hydrogen Bond Research, Monatshefte fûr Chemie, Chemical Monthly, vol. 130, Springer, New York, NY, USA, 8th edition, 1999.

[5] D. Hadzi, Ed., Theoretical Treatments of Hydrogen Bonding, Wiley, New York, NY, USA, 1997.

[6] A. Witkowski, "Infrared spectra of the hydrogen-bonded carboxylic acids," The Journal of Chemical Physics, vol. 47, no. 9, pp. 3679-3680, 1967.

[7] Y. Marechal and A. Witkowski, "Infrared spectra of H-bonded systems," The Journal of Chemical Physics, vol. 48, no. 8, pp. 3697-3705, 1968.

[8] S. F. Fischer, G. L. Hofacker, and M. A. Ratner, "Spectral behavior of hydrogen-bonded systems: quasiparticle model," The Journal of Chemical Physics, vol. 52, no. 4, pp. 1934-1947, 1970.

[9] O. Henri-Rousseau and P. Blaise, "The infrared spectral density of weak hydrogen bonds within the linear response theory," Advances in Chemical Physics, vol. 103, pp. 1-137, 1998.

[10] O. Henri-Rousseau and P. Blaise, "The $\mathrm{V}_{\mathrm{X}-\mathrm{H}}$ line shapes of centrosymmetric cyclic dimers involving weak hydrogen bonds," Advances in Chemical Physics, vol. 139, pp. 245-496, 2008.

[11] M. J. Wójcik, "Theoretical interpretation of infrared spectra of the $\mathrm{Cl}-\mathrm{H}$ stretching vibration in the gaseous $(\mathrm{Ch} 3) 2 \mathrm{O} \cdots \mathrm{HCl}$ complex," International Journal of Quantum Chemistry, vol. 29, no. 4, pp. 855-865, 1986.

[12] J. L. Leviel and Y. Marechal, "Infrared spectra of H-bonded systems: anharmonicity of the H-bond vibrations in cyclic dimers," The Journal of Chemical Physics, vol. 54, no. 3, pp. 1104-1107, 1971.
[13] J. Bournay and Y. Maréchal, "Dynamics of protons in hydrogen-bonded systems: propynoic and acrylic acid dimers," The Journal of Chemical Physics, vol. 55, no. 3, pp. 1230-1235, 1971.

[14] P. Excoffon and Y. Marechal, "Infrared spectra of H-bonded systems: saturated carboxylic acid dimers," Spectrochimica Acta A, vol. 28, no. 2, pp. 269-283, 1972.

[15] M. J. Wójcik, "Theory of the infrared spectra of the hydrogen bond in molecular crystals," International Journal of Quantum Chemistry, vol. 10, no. 4, pp. 747-760, 1976.

[16] H.T. Flakus and A. Bańczyk, "Abnormal distribution of protons and deuterons between the hydrogen bonds in cyclic centrosymmetric dimers in partially deuterated samples," Journal of Molecular Structure, vol. 476, no. 1-3, pp. 57-68, 1999.

[17] H. T. Flakus, "Vibronic model for H/D isotopic self-organization effects in centrosymmetric dimers of hydrogen bonds," Journal of Molecular Structure, vol. 646, no. 1-3, pp. 15-23, 2003.

[18] H. T. Flakus and A. Michta, "Investigations of interhydrogen bond dynamical coupling effects in the polarized IR spectra of acetanilide crystals," Journal of Physical Chemistry A, vol. 114, no. 4, pp. 1688-1698, 2010.

[19] R. W. G. Wyckoff, Crystal Structures, vol. 5, Wiley, New York, NY, USA, 1972.

[20] Z. Berkovitch-Yellin and L. Leiserowitz, "Atom-atom potential analysis of the packing characteristics of carboxylic acids. A study based on experimental electron density distributions," Journal of the American Chemical Society, vol. 104, no. 15, pp. 4052-4064, 1982.

[21] E. B. Wilson, J. C. Decius, and P. C. Cross, Molecular Vibrations; The Theory of Infrared and Raman Vibrational Spectra, McGraw- Hill, New York, NY, USA, 1955.

[22] H. T. Flakus and A. Miros, "Infrared spectra of the hydrogen bonded glutaric acid crystals: polarization and temperature effects," Journal of Molecular Structure, vol. 484, no. 1-3, pp. 103-115, 1999.

[23] H. T. Flakus and M. Chelmecki, "Infrared spectra of the hydrogen bond in benzoic acid crystals: temperature and polarization effects," Spectrochimica Acta A, vol. 58, no. 1, pp. 179-196, 2002.

[24] H. T. Flakus and M. Jabłońska, "Study of hydrogen bond polarized IR spectra of cinnamic acid crystals," Journal of Molecular Structure, vol. 707, no. 1-3, pp. 97-108, 2004.

[25] H. T. Flakus and M. Chełmecki, "Polarization IR spectra of the hydrogen bond in phenylacetic acid crystals: H/D isotopic effects-temperature and polarization effects," Spectrochimica Acta Part A, vol. 58, no. 9, pp. 1867-1880, 2002.

[26] H. T. Flakus and M. Chełmecki, "Polarization IR spectra of hydrogen bonded 1-naphthoic acid and 2-naphthoic acid crystals: electronic effects in the spectra," Journal of Molecular Structure, vol. 659, no. 1-3, pp. 103-117, 2003.

[27] H. T. Flakus and M. Chełmecki, "Polarization IR spectra of the hydrogen bond in 1-naphthylacetic and 2-naphthylacetic acid crystals: H/D isotopic effects. Temperature and polarization effects," Journal of Molecular Structure, vol. 705, no. 1-3, pp. 81-89, 2004.

[28] S. E. Filippakis and G. M. J. Schmidt, "Topochemistry. Part XVI. The crystal structure of trans- $\beta$-2-furylacrylic acid," Journal of the Chemical Society B, pp. 229-232, 1967.

[29] M. Danish, S. Ali, M. Mazhar, A. Badshah, and E. R. T. Tieking, "Crystal structure of 3-(2-Furyl)acrylic Acid, $\mathrm{C}_{7} \mathrm{H}_{6} \mathrm{O}_{3}$," Zeitschrift für Kristallographie, vol. 210, no. 9, p. 703, 1995. 
[30] H. T. Flakus and A. Tyl, "Polarized IR spectra of the hydrogen bond in acetic acid crystals," Chemical Physics, vol. 336, no. 1, pp. 36-50, 2007.

[31] H. T. Flakus and B. Stachowska, "A systematic study of polarized IR spectra of the hydrogen bond in formic acid crystals," Chemical Physics, vol. 330, no. 1-2, pp. 231-244, 2006.

[32] A. Tyl, E. Chełmecka, M. Jabłońska et al., "X-ray analysis at $150 \mathrm{~K}$, synthesis and theoretical calculations of 1-naphthaleneacrylic acid," Structural Chemistry, vol. 23, no. 2, pp. 325-323, 2012.

[33] H. T. Flakus, M. Jabłońska, and P.G. Jones, "Study of polarized IR spectra of the hydrogen bond system in crystals of styrylacetic acid," Spectrochimica Acta A, vol. 65, no. 2, pp. 481-489, 2006.

[34] G. Fisher, Vibronic Coupling, Acadamic Press, London, UK, 1984.

[35] H. T. Flakus, "On the vibrational transition selection rules for the centrosymmetric hydrogen-bonded dimeric systems," Journal of Molecular Structure C, vol. 187, pp. 35-53, 1989.

[36] H. T. Flakus, A. Pyzik, A. Michta, and J. Kusz, "'Reversal' exciton coupling effect in the IR spectra of the hydrogen bond cyclic dimers; polarized IR spectra of 3-hydroxy-4-methyl$2(3 \mathrm{H})$-thiazolethione crystals," Vibrational Spectroscopy, vol. 44, no. 1, pp. 108-120, 2007.

[37] H. T. Flakus and A. Tyl, "Strong vibrational exciton coupling effects in polarized IR spectra of the hydrogen bond in 2thiopyridone crystals," Vibrational Spectroscopy, vol. 47, no. 2, pp. 129-138, 2008.

[38] H. T. Flakus, A. Tyl, and A. Maślankiewicz, "Electron-induced phase transition in hydrogen-bonded solid-state 2-pyridone," Journal of Physical Chemistry A, vol. 115, no. 6, pp. 1027-1039, 2011.

[39] H. T. Flakus, A. Miros, and P. G. Jones, "Influence of molecular electronic properties on the IR spectra of dimeric hydrogen bond systems: polarized spectra of 2-hydroxybenzothiazole and 2-mercaptobenzothiazole crystals," Journal of Molecular Structure, vol. 604, no. 1, pp. 29-44, 2002.

[40] H. T. Flakus and A. Machelska, "Polarization IR spectra of hydrogen bonded pyrazole crystals: self-organization effects in proton and deuteron mixture systems. Long-range H/D isotopic effects," Spectrochimica Acta Part A, vol. 58, no. 314, pp. 553-566, 2002.

[41] H. T. Flakus, A. Tyl, and P. G. Jones, “'Self-organization' processes in proton and deuteron mixtures in open-chain hydrogen bond systems: Polarization IR spectra of 4-mercaptopyridine crystals," Spectrochimica Acta A, vol. 58, no. 2, pp. 299 $310,2002$.

[42] H. T. Flakus, W. Śmiszek-Lindert, and K. Stadnicka, "Strong vibronic coupling effects in polarized IR spectra of the hydrogen bond in N-methylthioacetamide crystals," Chemical Physics, vol. 335, no. 2-3, pp. 221-232, 2007.

[43] C. A. Davydov, Teorya Molekularnykh Ekscitonov, Nauka, Moscow, Russia, 1968.

[44] R. L. Hochstrasser, Molecular Aspects of Symmetry, W. A. Benjamin Inc., New York, NY, USA, 1966.

[45] L. A. Nafie, "Adiabatic molecular properties beyond the BornOppenheimer approximation. Complete adiabatic wave functions and vibrationally induced electronic current density," The Journal of Chemical Physics, vol. 79, no. 10, pp. 4950-4957, 1983.

[46] H.T. Flakus, "The effect of strong coupling between vibrations in hydrogen bonds on the polarized spectra of the mercaptobenzothiazole crystal: an "anomalous" isotopic effect," Chemical Physics, vol. 62, no. 1-2, pp. 103-114, 1981.
[47] P. Blaise, M. J. Wojcik, and O. Henri-Rousseau, "Theoretical Interpretation of the Lineshape of the Gaseous Acetic Acid Dimer.," Journal of Chemical Physics, vol. 122, Article ID 064306, 2005.

[48] N. Rekik, H. Ghalla, H. T. Flakus, M. Jablońska, P. Blaise, and B. Oujia, "Polarized infrared spectra of the H(D) bond in 2thiophenic acid crystals: a spectroscopic and computational study," ChemPhysChem, vol. 10, no. 17, pp. 3021-3033, 2009.

[49] R. Najeh, G. Houcine, H. T. Flakus, M. Jablonska, and O. Brahim, "Experimental and theoretical study of the polarized infrared spectra of the hydrogen bond in 3-thiophenic acid crystal," Journal of Computational Chemistry, vol. 31, no. 3, pp. 463-475, 2010.

[50] H. T. Flakus, N. Rekik, and A. Jarczyk, "Polarized IR spectra of the hydrogen bond in 2-thiopheneacetic acid and 2-thiopheneacrylic acid crystals: H/D isotopic and temperature effects," The Journal of Physical Chemistry A, vol. 116, no. 9, pp. 21172130, 2012.

[51] L. J. Bellamy and P. E. Rogasch, "Proton transfer in hydrogen bonded systems," Proceedings of the Royal Society A, vol. 257, pp. 98-108, 1960.

[52] H. T. Flakus, A. Michta, M. Nowak, and J. Kusz, "Effects of dynamical couplings in IR spectra of the hydrogen bond in N-phenylacrylamide crystals," Journal of Physical Chemistry A, vol. 115 , no. 17 , pp. 4202-4213, 2011. 

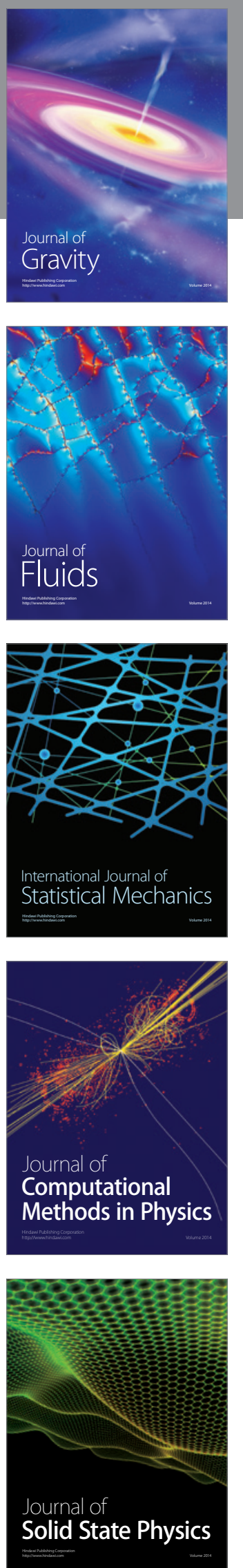

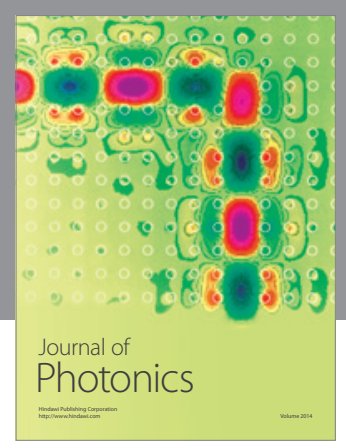

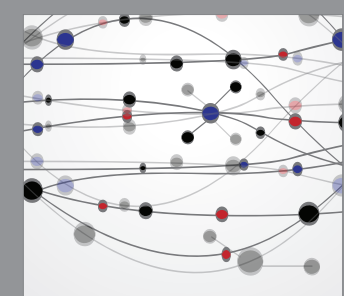

The Scientific World Journal
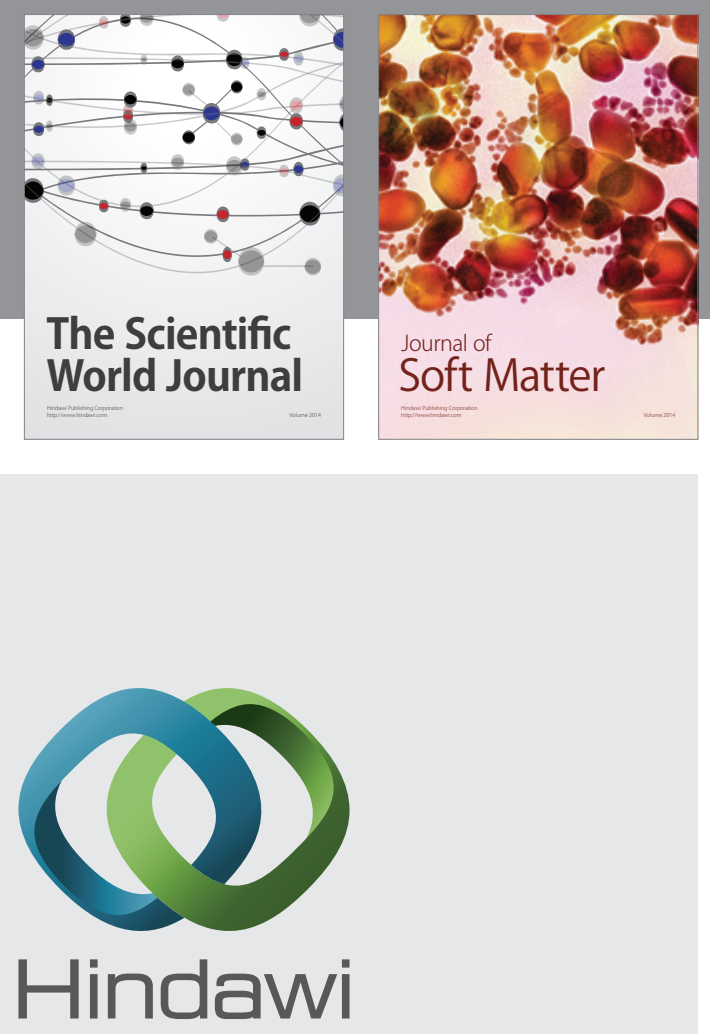

Submit your manuscripts at

http://www.hindawi.com
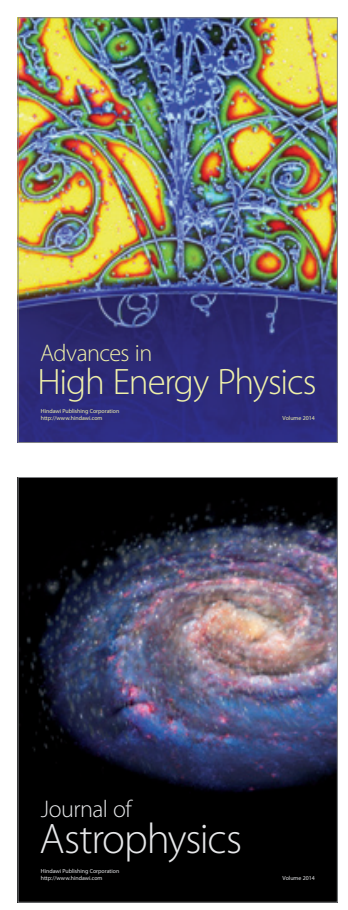
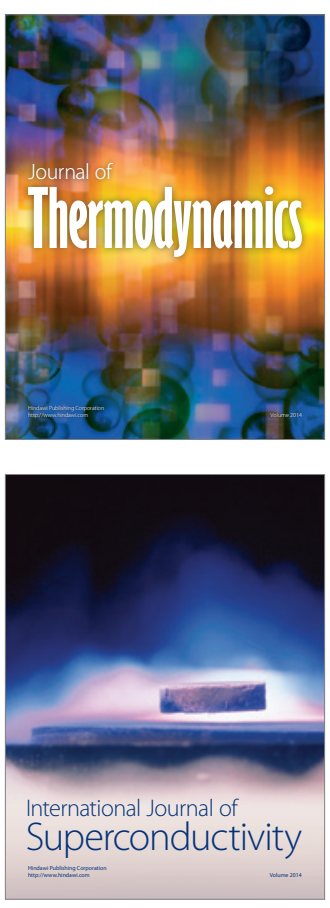
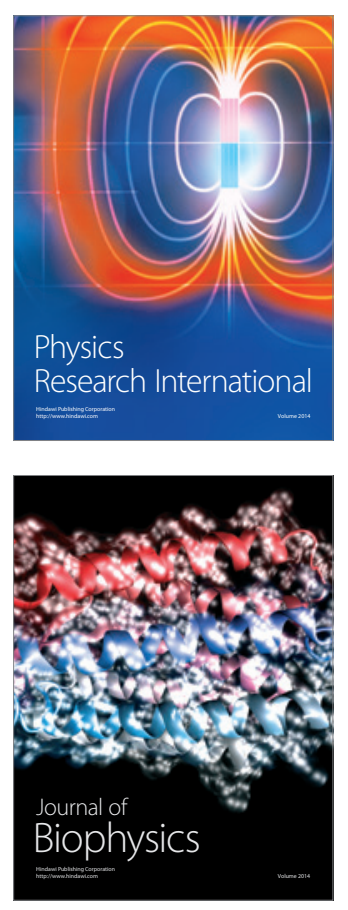
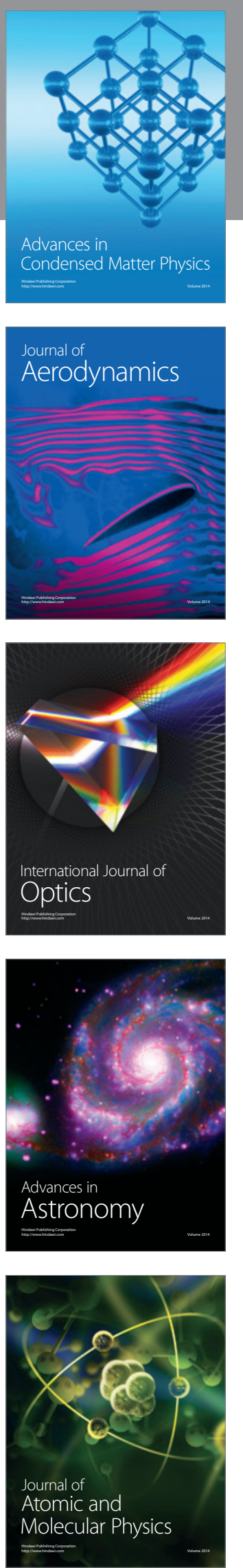